\title{
Spectroscopic determination of the fundamental parameters of 66 B-type stars in the field-of-view of the CoRoT satellite
}

\author{
K. Lefever ${ }^{1,2}$, J. Puls ${ }^{3}$, T. Morel ${ }^{1,4}$, C. Aerts ${ }^{1,5}$, L. Decin ${ }^{1, \star}$, and M. Briquet ${ }^{1, \star}$ \\ 1 Instituut voor Sterrenkunde, K.U. Leuven, Celestijnenlaan 200D, 3001 Leuven, Belgium \\ e-mail: Karolien.Lefever@aeronomie.be \\ 2 BIRA-IASB, Ringlaan 3, 1180 Brussel, Belgium \\ Universitätssternwarte München, Scheinerstr. 1, 81679 München, Germany \\ 4 Institut d'Astrophysique et de Géophysique, Université de Liège, Allée du 6 Août 17, Bât B5c, 4000 Liège, Belgium \\ 5 Departement Astrofysica, IMAPP, Radboud Universiteit Nijmegen, PO Box 9010, 6500 GL Nijmegen, The Netherlands
}

Received 26 February 2009 / Accepted 11 October 2009

\begin{abstract}
Aims. We determine the fundamental parameters of a sample of B stars with apparent visual magnitudes brighter than 8 in the fieldof-view of the CoRoT space mission, from high-resolution spectroscopy.

Methods. We developed an automatic procedure for the spectroscopic analysis of B-type stars with winds, based on an extensive grid of FASTWIND model atmospheres. We use the equivalent widths and/or the line profile shapes of continuum normalized hydrogen, helium, and silicon line profiles to determine the fundamental properties of these stars in an automated way.

Results. After thorough tests, on both synthetic datasets and very high-quality, high-resolution spectra of B stars for which we already had accurate values of physical properties from alternative analyses, we applied our method to 66 B-type stars contained in the ground-based archive of the CoRoT space mission. We discuss the statistical properties of the sample and compare them with those predicted by evolutionary models of B stars.

Conclusions. Our spectroscopic results provide a valuable starting point for any future seismic modelling of the stars, should they be observed by CoRoT.
\end{abstract}

Key words. stars: atmospheres - stars: early-type - stars: fundamental parameters - methods: data analysis techniques: spectroscopic - line: profiles

\section{Introduction}

The detailed spectroscopic analysis of B-type stars has for a long time been restricted to a limited number of targets. The reasons for this are the a priori need for a realistic atmosphere model, the lack of large samples with high-quality spectra, and the laborious process of line-profile fitting, since the multitude of photospheric and wind parameters require a large parameter space to be explored.

The advent of high-resolution, high signal-to-noise spectroscopy led to renewed interest in spectroscopic research, and in particular the relatively poorly understood massive stars. The establishment of continuously superior instrumentation and the improvement in quality of the spectroscopic data obtained triggered a series of studies, which led to a rapid enhancement in our knowledge of massive stars. In this respect, it is not surprising to note that this is exactly the period where several groups started to upgrade their atmosphere prediction code for these stars (see, e.g., CMFGEN - Hillier \& Miller 1998, PHOENIX - Hauschildt \& Baron 1999, WM-Basic - Pauldrach et al. 2001, POWR Gräfener et al. 2002, and FASTWIND - Santolaya-Rey et al. 1997, Puls et al. 2005). Initially, much attention was devoted to the establishment of a realistic atmosphere model (i.e. improvement of atomic data, inclusion of line blanketing and clumping), rather than analyzing large samples of stars.

$\star$ Postdoctoral researcher of the Fund for Scientific Research of Flanders (FWO).
In addition to improvements in the atmosphere predictions, the available amount of high-quality data has increased rapidly, mainly because of the advent of multi-object spectroscopy. At the time of writing, the largest survey is the VLT-FLAMES survey of massive stars (Evans et al. 2005), which contains over 600 Galactic, SMC, and LMC B-type spectra ${ }^{1}$ (in seven different clusters), gathered over more than $100 \mathrm{~h}$ of VLT time. The survey has not only allowed us to derive the stellar parameters and rotational velocities of hundreds of stars (Dufton et al. 2006; Hunter et al. 2008b), but also to study the evolution of surface $\mathrm{N}$ abundances and the effective temperature scales in the Galaxy and Magellanic Clouds (Trundle et al. 2007; Hunter et al. 2007, 2008a).

In preparation for the CoRoT space mission, and almost contemporary with the FLAMES setup, another large database was constructed: Ground-based Asteroseismology Uniform Database Interface (GAUDI) (Solano et al. 2005). It gathers ground-based observations of more than 1500 objects, including high-resolution spectra of about 250 massive B-type stars, with the goal of determining their fundamental parameters as input to seismic modeling (see Sect. 3).

The availability of these large samples of B-type stars enables different types of studies to be performed, e.g., leading to a significant improvement in the fundamental parameter calibration for this temperature range and to a confrontation with and evaluation of stellar evolution models (e.g., Hunter et al. 2008b). The drawback of this enormous amount of data, however, is,

\footnotetext{
${ }^{1}$ Additionally, roughly 90 O-stars have been observed.
} 
as mentioned before, the large parameter space that must be explored, a time-consuming process, if no adequate method is available. It requires a method that is able to derive the complete set of parameters of stars with a wide variety of physical properties in an objective way.

To deal with the large GAUDI dataset, we investigated the possibility of automated spectral line fitting and opted for a gridbased fitting method: AnalyseBstar. In Sect. 2, we justify our choice of a grid-based method, present the setup and discuss several tests which were applied to check the performance of the routine. A more detailed description of our methodology can be found in the appendix. Section 3 illustrates the first application of AnalyseBstar to the sample of CoRoT candidate targets in the GAUDI database and Sect. 4 deals with the physical interpretation and some statistical properties of the resulting parameters. Section 5 summarizes the main results obtained in this paper.

\section{Automated fitting using a grid-based method}

Spectral line fitting is a clear example of an optimization problem. To determine the optimal fit for a given observed stellar spectrum among a set of theoretically predicted spectra from stellar atmosphere models, we scan the space defined by the free parameters of the stellar atmosphere model. Because of the size of this parameter space and in terms of analyzing large samples, it is clear that performing the parameter scan by means of fitby-eye is inappropriate. The "subjective" eye should be replaced by an "intelligent" algorithm, in such way that the procedure of finding the optimum fit becomes automatic, objective, fast, and reproducible, even though human intervention can never be excluded completely.

\subsection{General description of our grid-based method}

In contrast to the case for $\mathrm{O}$ stars (e.g., Mokiem et al. 2005), treating the entire spectral range $\mathrm{B}$ with a single approach requires additional diagnostic lines besides $\mathrm{H}$ and $\mathrm{He}$. In this region, $\mathrm{Si}$ (in its different ionization stages), rather than $\mathrm{He}$, becomes the most appropriate temperature indicator. With this in mind, we chose to develop an automatic procedure based on an extensive and refined grid of FASTWIND models. This offers a good compromise between effort, time, and precision if an appropriate grid has been set up. The grid should be comprehensive, as dense as possible, and representative of the kind of objects one wishes to analyze. As for the fit-by-eye method, a grid-based algorithm will follow an iterative scheme, but in a reproducible way using a goodness-of-fit parameter. Starting from a first guess for the fundamental parameters, based on spectral type and/or published information, improved solutions are derived by comparing line profiles resulting from well-chosen existing (i.e., pre-calculated) grid models to the observed line profiles. The algorithm terminates once the fit quality cannot be improved anymore by modifying the model parameters.

We are aware of the limitations inherent to this method. When a new version/update of the atmosphere or line synthesis code is released (e.g., due to improved atomic data), the grid needs to be updated as well. An advantage, on the other hand, is that the line-profile fitting method itself remains unchanged and the revision involves only the computation of a new grid. This is an enormous amount of work (e.g., seven months were needed to compute $^{2}$ and check the grid in Lefever et al. 2007a), but because

\footnotetext{
2180 CPU months were needed to compute the full grid of almost 265000 models. To reduce the effective computation time, calculations
}

of the efficient performance of the FASTWIND code, this should not be an insurmountable problem.

A grid-based method fully relies on a static grid and no additional models are computed to derive the most likely parameters. Consequently, the quality of the final best fit and the precision of the final physical parameters are fully determined by the density of the grid. This underlines, again, the necessity of having a grid that is as dense as possible, allowing for interpolation. On the other hand, the grid method has a plus-point: it is fast. Whereas the analysis with, e.g., a genetic algorithm approach (e.g., Mokiem et al. 2005) needs parallel processors and several days of CPU to model one target star, our grid method will, on average, require less than half an hour on one computer, because no additional model computations are required.

\subsection{The code AnalyseBstar}

Our grid-based code, called AnalyseBstar, was developed for the spectroscopic analysis of B stars with winds. It is written in the Interactive Data Language (IDL), which allows for interactive manipulation and visualization of data. It fully relies on the extensive grid of NLTE model atmospheres and the emerging line profiles presented in Lefever et al. (2007a, see also Appendix B.8 for a brief overview of the considered parameter set) and is developed to treat large samples of stars in a homogeneous way. We use continuum-normalized $\mathrm{H}, \mathrm{He}$, and $\mathrm{Si}$ lines to derive the photospheric properties of the star and the characteristics of its wind. This automated method is not only homogeneous, but also objective and robust. A full description of the design of AnalyseBstar, including the preparation of the input, the iteration cycle for the determination of each physical parameter, and the inherent assumptions, is given in Appendix $\mathrm{B}^{3}$.

The intrinsic nature of our procedure allows us to derive estimates of the true parameters of the star, which in most cases are in-between those of two model grid points. In what follows, we refer to them as "interpolated values". The corresponding grid values are the parameters corresponding to the grid model lying closest to these interpolated values.

Furthermore, we denote the surface gravity as being derived from fitting the Balmer line wings as $\log g$, while the gravity corrected for centrifugal terms (required, e.g., to estimate consistent masses) will be denoted by $\log g_{c}$. The (approximate) correction itself is obtained by adding the term $(v \sin i)^{2} / R_{*}$ to the uncorrected gravity (Repolust et al. 2005, and references therein), with $v \sin i$ the projected rotational velocity.

\subsection{Testing the method}

\subsubsection{Convergence tests for synthetic FASTWIND spectra}

Before applying AnalyseBstar to observed stellar spectra, we tested whether the method was able to recover the parameters of synthetic FASTWIND input spectra. To this end, we created several synthetic datasets in various regions of parameter space, with properties representative of a "typical" GAUDI spectrum.

were done on a dedicated Linux cluster of 5 dual-core, dual-processor computers $(3800 \mathrm{MHz}$ processors, sharing $4 \mathrm{~Gb}$ RAM memory and $8 \mathrm{~Gb}$ swap memory), amounting to 20 dedicated CPU processors, in addition to 40 more regularly used institute CPUs ( 8 of $3800 \mathrm{MHz}$ and 32 of $3400 \mathrm{MHz}$ ). The grid filled $60 \%$ of a terabyte disk, connected to a Solaris 10 host pc.

${ }^{3}$ Manual available upon request from the authors. 
For more details of the setup of this test dataset and the results of the convergence tests, we refer to Appendix A. In all cases, the input parameters were recovered well and no convergence problems were encountered. Minor deviations from the input parameters were found to be as expected and within the errorbars. Also the derived $v \sin i$-values, which are difficult to differentiate from potential macroturbulent velocities $\left(v_{\text {macro }}\right)$, agreed very well with the inserted values, irrespective of $v_{\text {macro }}$. This shows that the Fourier transform method of Gray (1973, 1975), whose implementation by Simón-Díaz \& Herrero (2007) we used, indeed allows us to separate both effects (but see below). Altogether, this provides us with enough confidence to believe that our procedure will also be able to recover the physical parameters from real spectral data.

\subsubsection{FASTWIND versus Tlusty: profile comparison and analysis of Tlusty spectra}

As an additional test, we compared line profiles based on FASTWIND models with negligible mass loss with their corresponding counterpart in the grid computed with the NLTE model atmosphere code Tlusty (Hubeny \& Lanz 2000), which also incorporates a fully consistent NLTE metal line blanketing. This resulted in overall good agreement, except for low gravity stars, where differences arise. It concerns differences in the forbidden He I components (which are quire strong in FASTWIND, but almost absent in Tlusty), the Balmer line wings (which are slightly stronger in FASTWIND than in Tlusty, yielding slightly lower $\log g$ values) and the Si II/III EW ratio for effective temperatures $T_{\text {eff }}>18 \mathrm{kK}$ (which is higher for FASTWIND than for Tlusty - due to both stronger Si II and weaker Si III lines -, yielding higher $T_{\text {eff }}$ values). The reason that FASTWIND predicts stronger Balmer line wings is differences in the broadening functions and an underestimated photospheric line pressure, which can affect the photospheric structure of low-gravity objects. Differences are about or below 0.1 dex, and become negligible for dwarf stars. Apart from a the problem with the forbidden components, the profiles of the cooler models $\left(T_{\text {eff }}<18 \mathrm{kK}\right)$ compare very well.

To check the uncertainty in the synthetic profiles, we applied AnalyseBstar to profiles of six prototypical Tlusty models at 3 different temperature points $(15000,20000$, and $25000 \mathrm{~K})$ and at a high and a low gravity. We added artificial noise to the data to obtain a typical SNR of 150 and artificially broadened the profiles with a fixed projected rotational velocity of $v \sin i=50 \mathrm{~km} \mathrm{~s}^{-1}$. The results of the comparison can be found in Table 1. The input and output fundamental parameters agree very well within the resulting errors, with only one exception, which is the model with high $T_{\text {eff }}$ and higher $\log g$, where the temperature is off by $2000 \mathrm{~K}$.

Around $20 \mathrm{kK}$, we experience similar difficulties in retrieving a unique temperature as in the cool B star domain, i.e., depending on the gravity, we may have only one stage of Si available, in this case Si III. Indeed, whereas the Si II lines are clearly visible in the higher gravity test case $(\log g=3.00)$, they are no longer detectable in the lower gravity case $(\log g=2.25)$. At this $T_{\text {eff }}$, there are (unfortunately) no obvious Si IV lines yet, which complicates the analysis. Even though, AnalyseBstar is still able to retrieve the correct effective temperature, albeit with larger errors, using the same alternative method developed for late B type stars when only Si II is available (see "method 2" in Sect. B.3).
Table 1. Result of applying AnalyseBstar to synthetic Tlusty profiles.

\begin{tabular}{cccc}
\hline \hline Input TLusty & & Output FASTWIND & (Best-fit model) \\
\hline$T_{\text {eff, in }}$ & $\begin{array}{c}\log g_{\text {,in }} \\
(\mathrm{cgs})\end{array}$ & $\begin{array}{c}T_{\text {eff,out }} \pm \Delta \\
(\mathrm{kK})\end{array}$ & $\begin{array}{c}\log g_{\text {,out }} \pm \Delta \\
(\mathrm{cgs})\end{array}$ \\
\hline 15 & 1.75 & $15.0 \pm 0.5$ & $1.7 \pm 0.05$ \\
15 & 3.00 & $15.5 \pm 0.5$ & $3.0 \pm 0.10$ \\
20 & 2.25 & $20.0 \pm 1.0$ & $2.2 \pm 0.10$ \\
20 & 3.00 & $21.0 \pm 1.0$ & $3.2 \pm 0.20$ \\
25 & 2.75 & $25.0 \pm 1.0$ & $2.7 \pm 0.05$ \\
25 & 3.00 & $27.0 \pm 1.0$ & $3.1 \pm 0.10$ \\
\hline
\end{tabular}

\subsubsection{Comparison with fit-by-eye results for well-studied pulsators}

Having thoroughly tested that AnalyseBstar indeed converges towards the optimal solution and that it is able to recover the input parameters of synthetic spectra, we performed an additional test on real spectra. We tested our method on a selected sample of high-quality, high-resolution spectra of pulsating B stars $(\beta$ Cephei and slowly pulsating B stars (SPB)). The (mean) spectra of these stars have a very high signal-to-noise ratio (SNR), attained by the addition of a large number of individual exposures (see Morel et al. 2007). Given that we excluded binaries from our GAUDI sample (see Sect. 3.1), we also ignored $\theta$ Oph and $\beta$ Cru for the test sample. Moreover, V2052 Oph is chemically peculiar and was thus excluded, as well as V836Cen for which only one spectrum is available. Thus, we limited our test sample to those single stars in Morel et al. (2007) for which numerous high-quality spectra were available. Because of the high quality of these spectral time series, they are ideally suited to testing AnalyseBstar.

The $\beta$ Cephei stars and two of the SPB stars were analyzed in detail by Morel et al. (2006, 2008) and Briquet \& Morel (2007), respectively. These authors used the latest version of the NLTE line formation codes DETAIL and SURFACE (Giddings 1981; Butler \& Giddings 1985), in combination with plane-parallel, fully line-blanketed LTE Kurucz atmospheric models (ATLAS9, Kurucz 1993), to determine (by eye) the atmospheric parameters and element abundances of low-luminosity class objects with negligible winds. The outcome of the comparison with their results are summarized in Tables 2 a to $2 \mathrm{c}$, and in Figs. 1 and 2. Typical errors for $T_{\text {eff }}$ and $\log g$ are, respectively, $1000 \mathrm{~K}$ and 0.15 for the comparison data sets of Morel et al. $(2006,2008)$ and Briquet \& Morel (2007), which is somewhat more conservative than the errors adopted in this study. We adopt typical $1-\sigma$ errors of 0.10 dex for $\log g, 1000 \mathrm{~K}$ for $T_{\text {eff }}>20000 K$ and $T_{\text {eff }}<15000 \mathrm{~K}$, and $500 \mathrm{~K}$ for $15000 \mathrm{~K} \leq T_{\text {eff }} \leq 20000 \mathrm{~K}$. The error in the derived Si-abundance, $\log n(\mathrm{Si}) / n(\mathrm{H})$, was estimated to be 0.15 and 0.20 dex for objects above and below $15000 \mathrm{~K}$, respectively (see also Sect. B.3).

Although the effective temperatures derived from FASTWIND tend to be, on average, slightly below the ones derived from DETAIL/SURFACE, it is clear that, within the errorbars, FASTWIND and DETAIL/SURFACE provide consistent results for $T_{\text {eff }}$ and $\log g$ (Fig. 1) as well as for $\log n(\mathrm{Si}) / n(\mathrm{H})$ (Fig. 2), On the other hand, the $v \sin i$ values derived by Morel et al. (2008) are typically slightly above those derived by us. This is readily understood because their values implicitly include the macroturbulent velocity, which has not been taken into account as a separate broadening component. In those cases where we derive a vanishing $v_{\text {macro }}$, the $v \sin i$ values are in perfect agreement. Aerts et al. (2009) suggested that macroturbulence might be explained in terms of collective 
Table 2a. Fundamental parameters of well-studied $\beta$ Cephei stars (spectral types from SIMBAD), as derived from AnalyseBstar (values from the closest grid model are listed).

\begin{tabular}{|c|c|c|c|c|c|c|c|c|c|}
\hline $\begin{array}{r}\text { HD } \\
\text { number }\end{array}$ & $\begin{array}{l}\text { Alternative } \\
\text { name }\end{array}$ & $\begin{array}{l}\text { Spectral } \\
\text { type }\end{array}$ & $\begin{array}{l}T_{\text {eff }} \\
(\mathrm{K})\end{array}$ & $\begin{array}{l}\log g^{a} \\
(\operatorname{cgs})\end{array}$ & $n(\mathrm{He}) / n(\mathrm{H})^{b}$ & $\log n(\mathrm{Si}) / n(\mathrm{H})^{c}$ & $\begin{array}{r}\xi \\
\left(\mathrm{km} \mathrm{s}^{-1}\right)\end{array}$ & $\begin{array}{c}v \sin i \\
\left(\mathrm{~km} \mathrm{~s}^{-1}\right)\end{array}$ & $\begin{array}{l}v_{\text {macro }} \\
\left(\mathrm{km} \mathrm{s}^{-1}\right)\end{array}$ \\
\hline \multirow[t]{2}{*}{46328} & $\xi^{1} \mathrm{CMa}$ & B0.5-B1IV & 27000 & 3.80 & 0.10 & -4.69 & 6 & $9 \pm 2$ & 11 \\
\hline & & & 27500 & 3.75 & & $-4.87 \pm 0.21$ & $6 \pm 2$ & $10 \pm 2$ & - \\
\hline \multirow[t]{2}{*}{50707} & $15 \mathrm{CMa}$ & B1III & 24000 & 3.40 & 0.10 & -4.79 & 12 & $34 \pm 4$ & 38 \\
\hline & & & 26000 & 3.60 & & $-4.69 \pm 0.30$ & $7 \pm 3$ & $45 \pm 3$ & - \\
\hline \multirow[t]{2}{*}{205021} & $\beta$ Cер & B1IV & 25000 & 3.80 & 0.10 & -4.70 & 6 & $26 \pm 3$ & 24 \\
\hline & & & 26000 & 3.70 & & $-4.89 \pm 0.23$ & $6 \pm 3$ & $29 \pm 2$ & - \\
\hline \multirow[t]{2}{*}{44743} & $\beta \mathrm{CMa}$ & B1.5III & 24000 & 3.50 & 0.10 & -4.76 & 15 & $19 \pm 4$ & 20 \\
\hline & & & 24000 & 3.50 & & $-4.83 \pm 0.23$ & $14 \pm 3$ & $23 \pm 2$ & - \\
\hline \multirow[t]{2}{*}{214993} & $12 \mathrm{Lac}$ & B $1.5 \mathrm{IV}$ & 23000 & 3.60 & 0.10 & $\begin{array}{l}-4.41 \\
{[-4.83]}\end{array}$ & $\begin{array}{r}6 \\
{[12]}\end{array}$ & $44 \pm 6$ & $\begin{array}{l}37 \\
{[0] \text { (forced) }}\end{array}$ \\
\hline & & & 24500 & 3.65 & & $-4.89 \pm 0.27$ & $10 \pm 4$ & $42 \pm 4$ & - \\
\hline \multirow[t]{2}{*}{16582} & $\delta$ Ceti & B1.5-B2IV & 23000 & 3.90 & 0.10 & -4.80 & 6 & $14 \pm 2$ & 0 \\
\hline & & & 23000 & 3.80 & & $-4.72 \pm 0.29$ & $1_{-1}^{+3}$ & $14 \pm 1$ & - \\
\hline \multirow[t]{2}{*}{886} & $\gamma$ Peg & B1.5-B2IV & 23000 & 3.80 & 0.10 & -4.84 & $<3$ & $10 \pm 1$ & 0 \\
\hline & & & 22500 & 3.75 & & $-4.81 \pm 0.29$ & $1_{-1}^{+2}$ & $10 \pm 1$ & - \\
\hline \multirow[t]{2}{*}{29248} & $v$ Eri & B1.5-B2IV & 23000 & 3.70 & 0.10 & -4.73 & 10 & $21 \pm 3$ & 39 \\
\hline & & & 23500 & 3.75 & & $-4.79 \pm 0.26$ & $10 \pm 4$ & $36 \pm 3$ & - \\
\hline
\end{tabular}

Notes. In italics, we display the results from the DETAIL/SURFACE analysis by Morel et al. (2006, 2008). ${ }^{(a)}$ Not corrected for centrifugal acceleration due to stellar rotation. ${ }^{(b)}$ Solar helium abundance: $n(\mathrm{He}) / n(\mathrm{H})=0.10 .{ }^{(c)}$ Solar silicon abundance: $\log n(\mathrm{Si}) / n(\mathrm{H})=-4.49$. Interpolated values are listed.

Table 2b. Same as for Table 2a, but now for some well-studied SPBs.

\begin{tabular}{rlllllcrcc}
\hline \hline $\begin{array}{r}\text { HD } \\
\text { number }\end{array}$ & $\begin{array}{l}\text { Alternative } \\
\text { name }\end{array}$ & $\begin{array}{l}\text { Spectral } \\
\text { Type }\end{array}$ & $\begin{array}{c}T_{\text {eff }} \\
(\mathrm{K})\end{array}$ & $\begin{array}{l}\log g \\
(\mathrm{cgs})\end{array}$ & $n(\mathrm{He}) / n(\mathrm{H})$ & $\log n(\mathrm{Si}) / n(\mathrm{H})$ & $\begin{array}{r}\xi \\
\left(\mathrm{km} \mathrm{s}^{-1}\right)\end{array}$ & $\begin{array}{c}v \sin i \\
\left(\mathrm{~km} \mathrm{~s}^{-1}\right)\end{array}$ & $\begin{array}{c}v_{\text {macro }} \\
\left(\mathrm{km} \mathrm{s}^{-1}\right)\end{array}$ \\
\hline 3360 & $\zeta$ Cas & B2IV & 22000 & 3.80 & 0.10 & -4.76 & $<3$ & $18 \pm 2$ & 13 \\
& & $a$ & 22000 & 3.70 & & $-4.72 \pm 0.30$ & $1 \pm 1$ & $19 \pm 1$ & - \\
85953 & V335 Vel & B2IV & 20000 & 3.80 & 0.10 & -4.84 & 6 & $30 \pm 1$ & 20 \\
& & & 21000 & 3.80 & & $-4.75 \pm 0.30$ & $1 \pm 1$ & $29 \pm 2$ & - \\
3379 & 53 Psc & B2.5IV & 20000 & 4.30 & 0.10 & -4.73 & $<3$ & $48 \pm 8$ & 43 \\
74195 & o Vel & B3IV & 16500 & 3.70 & 0.10 & -4.79 & $<3$ & $18 \pm 2$ & 18 \\
160762 & $\iota$ Her & B3IV & 19500 & 4.10 & 0.10 & -4.86 & 3 & $8 \pm 2$ & 0 \\
25558 & 40 Tau & B3V & 17500 & 4.00 & 0.10 & -4.72 & 6 & $28 \pm 2$ & 31 \\
181558 & HR 7339 & B5III & 15000 & 4.00 & 0.10 & -4.79 & 6 & $17 \pm 2$ & 0 \\
26326 & HR 1288 & B5IV & 15500 & 3.60 & 0.10 & -4.49 & $<3$ & $17 \pm 1$ & 17 \\
206540 & HR 8292 & B5IV & 13500 & 3.80 & 0.10 & -4.79 & 3 & $15 \pm 2$ & 0 \\
24587 & HR 1213 & B5V & 14500 & 4.00 & 0.10 & -4.79 & $<3$ & $25 \pm 4$ & 21 \\
28114 & HR 1397 & B6IV & 14000 & 3.50 & 0.10 & -4.79 & $<3$ & $21 \pm 4$ & 17 \\
138764 & HR 5780 & B6IV & 14500 & 3.90 & 0.10 & -4.51 & 3 & $21 \pm 2$ & 0 \\
215573 & HR 8663 & B6IV & 13500 & 3.80 & 0.10 & -4.49 & $<3$ & $8 \pm 1$ & 0 \\
39844 & HR 2064 & B6V & 14500 & 3.70 & 0.10 & -4.39 & $<3$ & $16 \pm 1$ & 0 \\
191295 & V1473 Aql & B7III & 13000 & 3.70 & 0.10 & -4.79 & 3 & $16 \pm 1$ & 15 \\
21071 & V576 Per & B7V & 13500 & 3.70 & 0.10 & -4.79 & 3 & $22 \pm 1$ & 0 \\
37151 & V1179 Ori & B8V & 12500 & 3.80 & 0.10 & -4.79 & $<3$ & $20 \pm 2$ & 0 \\
\hline
\end{tabular}

Notes. ${ }^{(a)}$ Independent results of the DETAIL/SURFACE analysis by Briquet \& Morel (2007).

pulsational velocity broadening caused by the superposition of a multitude of gravity modes with low amplitudes (see also Appendix B.4). In this respect, we expect stars for which $v_{\text {macro }}$ differs from zero to be pulsators.

For a few overlapping objects, we could also compare our results with those from independent studies by Mokiem et al. (2005), based on FASTWIND predictions for $\mathrm{H}$ and He lines and using a genetic algorithm approach, and by Przybilla et al. (2008), using ATLAS9/DETAIL/SURFACE, allowing for a comparison of $\mathrm{H}, \mathrm{He}$, and $\mathrm{Si}$. The consistency with both studies is quite good, except for the Si abundance of HD 36591 determined by Przybilla et al. For this object, Hubrig et al. (2008) obtained a value close to ours, using the same
ATLAS9/DETAIL/SURFACE code. Ad hoc, we cannot judge the origin of this discrepancy.

For two targets, HD 85953 and $\tau$ Sco, somewhat larger differences between the derived microturbulence are found. For HD 85953, this difference is compensated by an opposite difference in the derived $\mathrm{Si}$ abundance. For $\tau \mathrm{Sco}$, on the other hand, the derived microturbulence (and also the Si abundance) is in perfect agreement with the values provided by Hubrig et al. (2008) and Przybilla et al. (2008) within the defined errorbars, whereas the value for the microturbulence derived by Mokiem et al. (2005) differs substantially. As they did not derive the Si abundance, we cannot assess on the difference in $\mathrm{Si}$ abundance this would have caused. 
Table 2c. Same as for Table 2a, but now for some well-studied hot B stars.

\begin{tabular}{|c|c|c|c|c|c|c|c|c|c|c|}
\hline $\begin{array}{r}\text { HD } \\
\text { number }\end{array}$ & $\begin{array}{l}\text { Alternative } \\
\text { name }\end{array}$ & $\begin{array}{l}\text { Spectral } \\
\text { Type }\end{array}$ & & $\begin{array}{l}T_{\text {eff }} \\
(\mathrm{K})\end{array}$ & $\begin{array}{l}\log g \\
\text { (cgs) }\end{array}$ & $n(\mathrm{He}) / n(\mathrm{H})$ & $\log n(\mathrm{Si}) / n(\mathrm{H})$ & $\begin{array}{r}\xi \\
\left(\mathrm{km} \mathrm{s}^{-1}\right)\end{array}$ & $\begin{array}{c}v \sin i \\
\left(\mathrm{~km} \mathrm{~s}^{-1}\right)\end{array}$ & $\begin{array}{l}v_{\text {macro }} \\
\left(\mathrm{km} \mathrm{s}^{-1}\right)\end{array}$ \\
\hline \multirow[t]{2}{*}{93030} & $\theta$ Car & B0Vp & & 31000 & 4.20 & 0.15 & -4.38 & 10 & $108 \pm 3$ & 0 \\
\hline & & & $a$ & 31000 & 4.20 & - & $-4.57 \pm 0.23$ & $12 \pm 4$ & $113 \pm 8$ & - \\
\hline \multirow[t]{4}{*}{149438} & $\tau \mathrm{Sco}$ & B $0.2 \mathrm{~V}$ & & 32000 & 4.20 & 0.10 & -4.55 & 6 & $10 \pm 2$ & 0 \\
\hline & & & $a$ & 31500 & 4.05 & - & $-4.76 \pm 0.14$ & $2 \pm 2$ & $8 \pm 2$ & - \\
\hline & & & $b$ & 31900 & 4.15 & 0.12 & - & 10.8 & 5 & - \\
\hline & & & $c$ & 32000 & 4.30 & 0.10 & -4.50 & 5 & 4 & 4 \\
\hline \multirow[t]{3}{*}{36591} & HR 1861 & B1V & & 26000 & 3.90 & 0.10 & -4.73 & 6 & $13 \pm 1$ & 0 \\
\hline & & & $d$ & 27000 & 4.00 & - & $-4.75 \pm 0.29$ & $3 \pm 2$ & $16 \pm 2$ & - \\
\hline & & & $c$ & 27000 & 4.12 & 0.10 & -4.52 & 3 & 12 & - \\
\hline \multirow[t]{2}{*}{52089} & $\epsilon \mathrm{CMa}$ & B1.5-B2II/I & & 22000 & 3.20 & 0.10 & -4.69 & 15 & $32 \pm 2$ & 20 \\
\hline & & & $d$ & $23000^{d}$ & 3.30 & - & $-4.77 \pm 0.24$ & $16 \pm 4$ & $28 \pm 2$ & - \\
\hline \multirow[t]{2}{*}{35468} & $\gamma$ Ori & B2II-III & & 22000 & 3.60 & 0.10 & -4.79 & 10 & $46 \pm 8$ & 37 \\
\hline & & & $d$ & 22000 & 3.50 & - & $-5.00 \pm 0.19$ & $13 \pm 5$ & $51 \pm 4$ & - \\
\hline \multirow[t]{2}{*}{51309} & $\iota \mathrm{CMa}$ & B2.5Ib-II & & 17000 & 2.60 & 0.10 & -4.78 & 15 & $27 \pm 4$ & 39 \\
\hline & & & $d$ & 17500 & 2.75 & - & $-4.82 \pm 0.31$ & $15 \pm 5$ & $32 \pm 3$ & - \\
\hline
\end{tabular}

Notes. ${ }^{(a)}$ Independent results of the DETAIL/SURFACE analysis by Hubrig et al. (2008). ${ }^{(b)}$ Values found by Mokiem et al. (2005) using a genetic algorithm approach. ${ }^{(c)}$ Results for two objects overlapping with the study of Przybilla et al. (2008). ${ }^{(d)}$ Independent results of the DETAIL/SURFACE analysis by Morel et al. (2006, 2008).
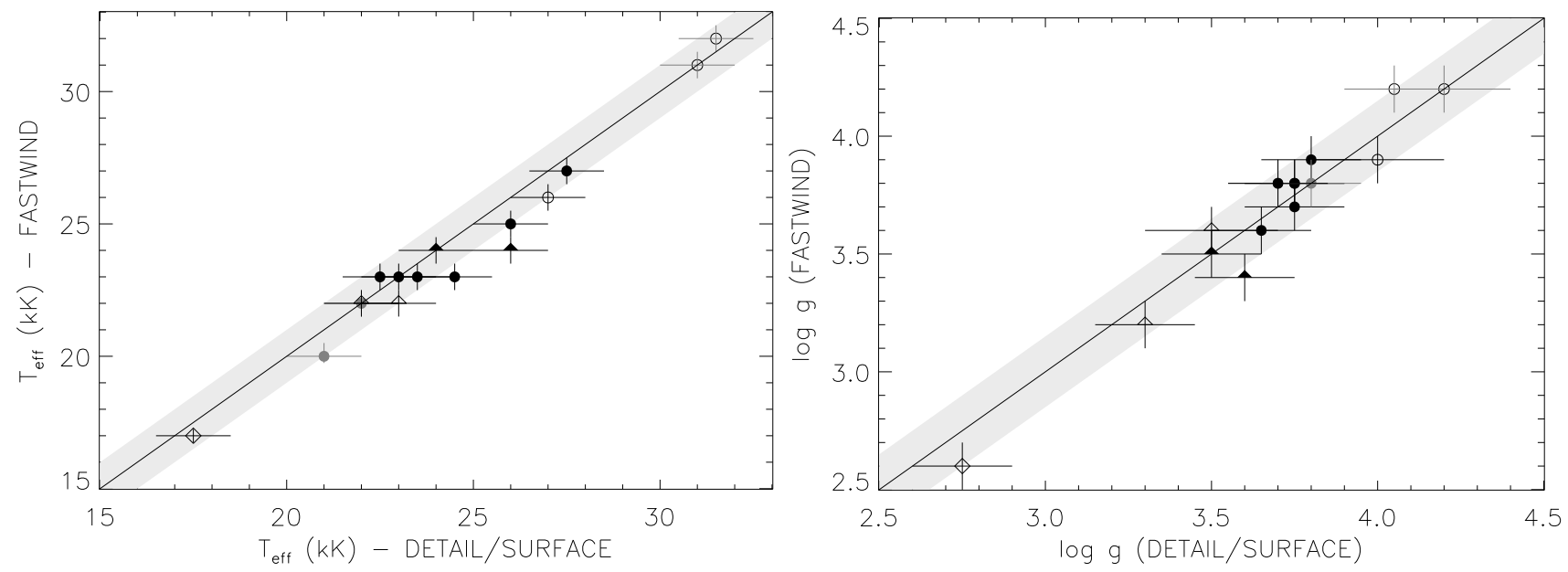

Fig. 1. Comparison of the effective temperatures (left) and surface gravities (right) derived with FASTWIND (AnalyseBstar) with those found by Morel et al. (2006, 2008) and Briquet \& Morel (2007) using DETAIL/SURFACE, for the $\beta$ Cephei stars (black), SPBs (grey) and some wellstudied hot B stars (open symbols). Supergiants are indicated as diamonds, giants as triangles, and dwarfs as circles. The shaded area around the one-to-one relation (straight line) denotes the uncertainty in the derived fundamental parameters of Morel et al. (2006, 2008) and Briquet \& Morel (2007) (i.e., $1000 \mathrm{~K}$ in $T_{\text {eff }}$ and 0.15 in $\log g$ ).

An even more detailed test can be completed for stars with seismically determined values of the fundamental parameters. $12 \mathrm{Lac}$ is such a star. It is known to be a non-radial pulsator with at least 11 independent oscillation frequencies (Handler et al. 2006). That $12 \mathrm{Lac}$ is a rich pulsator clearly shows up in the line profiles, which are strongly asymmetric. In particular, the Si III triplets (Si III 4552-4567-4574 and Si III 4813-4819-4829) are skew. Figure 3 shows the observed spectrum (black), which is a combination of 31 exposures. Using the Fourier transform method, we find the projected rotational velocity to be $44 \mathrm{~km} \mathrm{~s}^{-1}$, which is slightly above the $36 \mathrm{~km} \mathrm{~s}^{-1}$ derived from modelling the line profile variations (Desmet et al. 2009). Including macroturbulence in the spectral line fitting, we deduce the following parameters: $T_{\text {eff }}=23000 \mathrm{~K} \pm 1000 \mathrm{~K}, \log g=3.6 \pm 0.1$, $\xi=6 \pm 3 \mathrm{~km} \mathrm{~s}^{-1}$, and solar He and $\mathrm{Si}$ abundances (solid grey lines in Fig. 3). The derived surface gravity is in perfect agreement with the seismically determined value of $\log g$ (3.64-3.70) determined by Desmet et al. (2009).
Apart from the Si abundance, our results are also in agreement with Morel et al. (2006, see Table 2a), who derived $T_{\text {eff }}=$ $24500 \mathrm{~K} \pm 1000 \mathrm{~K}, \log g=3.65 \pm 0.15, \xi=10 \pm 4 \mathrm{~km} \mathrm{~s}^{-1}$, and depleted Si abundances as seem to be typical of B dwarfs in the solar neighborhood. To account for the pulsational broadening, we needed to include a macroturbulence of $37 \mathrm{~km} \mathrm{~s}^{-1}$. Forcing the macroturbulence to be zero while keeping the derived value of $v \sin i$ leaves the main physical parameters $\left(T_{\mathrm{eff}}, \log g\right.$ and He abundance) unaltered, while the microturbulent velocity $\xi$ and the $\mathrm{Si}$ abundance change to compensate for the change in profile shape: the microturbulence becomes larger $\left(12 \mathrm{~km} \mathrm{~s}^{-1}\right.$, broader lines) and the $\mathrm{Si}$ abundance becomes lower (depleted, $\log n(\mathrm{Si}) / n(\mathrm{H}) \approx-4.83)$. As expected, this depleted Si abundance $i s$ in agreement with Morel et al. (2006), who did not include $v_{\text {macro }}$ in their analysis. When evaluating the fit quality, we find, besides the expected mismatch in the Si line wings, also a discrepancy in the line cores of $\mathrm{He}$ (dashed grey lines in Fig. 3). Following Aerts et al. (2009), this might be improved 


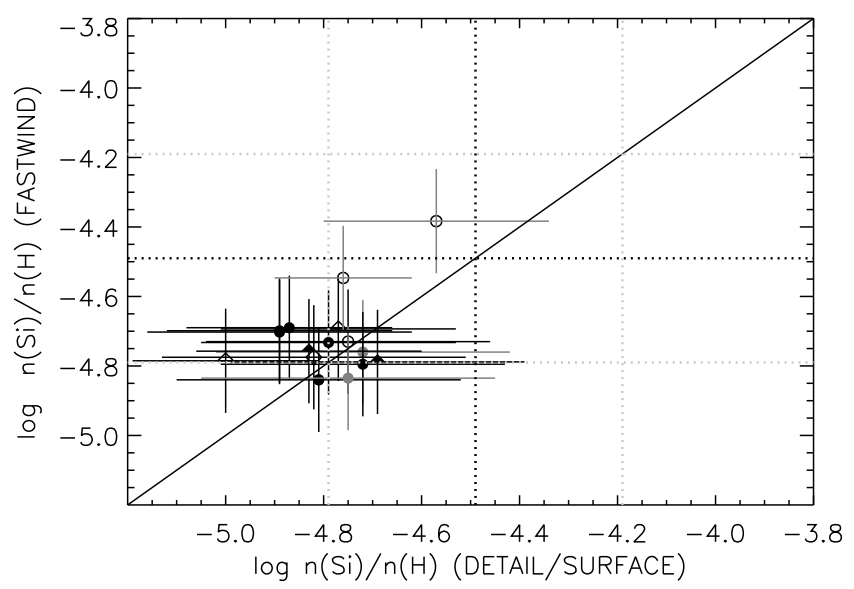

Fig. 2. Comparison between the $\mathrm{Si}$ abundances derived from AnalyseBstar and those derived from DETAIL/SURFACE for the set of photometric targets, used to evaluate the performance of AnalyseBstar. The black dotted lines represent the solar Si abundance $(\log n(\mathrm{Si}) / n(\mathrm{H})=-4.49)$, while the grey dotted lines represent a depletion and enhancement of Si by 0.3 dex (i.e., -4.79 and -4.19 , respectively). Symbols have the same meaning as in Fig. 1. Individual errorbars are indicated.

by accounting for the pulsational broadening introduced by the collective set of detected oscillations in $12 \mathrm{Lac}$, as this would result in a shape intermediate between a rotation and a Gaussian profile. However, the only way one can account for this, is by using an appropriate time series of spectra. The above example illustrates that we need to account for pulsational broadening to correctly assess the $\mathrm{Si}$ abundance and microturbulence. In this

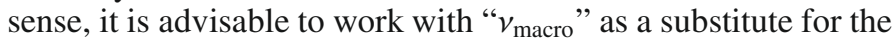
time-dependent broadening in the case of "snapshot" spectra.

\section{Application to the GAUDI B star sample}

\subsection{CoRoT \& GAUDI}

The French-led European space mission CoRoT (Convection, Rotation, and planetary Transits; see "the CoRoT Book", Fridlund et al. 2006) was launched successfully on December 27th, 2006. The observational setup of the CoRoT seismology programme (observations of a small number of bright stars over a very long period) made target selection a crucial issue. Because of the acute shortage of available information about the potential CoRoT targets, the need for additional data was high. Therefore, an ambitious ground-based observing program of more than 1500 objects was set up, under the leadership of C. Catala at Meudon, to obtain Strömgren photometry $(u v b y \beta)$ as well as high-resolution spectroscopy (FEROS, ELODIE, SARG, CORALIE, GIRAFFE, coudé spectrograph at the $2 \mathrm{~m}$ telescope in Tautenburg, CATANIA). These data were collected in an extensive catalogue, maintained at LAEFF (Laboratorio de Astrofísica Espacial y Física Fundamental) and baptized GAUDI: Ground-based Asteroseismology Uniform Database Interface (Solano et al. 2005).

The Be stars within the GAUDI database were studied by Frémat et al. (2006) and Neiner et al. (2005) and are omitted here, since the FASTWIND code is not developed to treat stars with a circumstellar disc. Also double-lined spectroscopic binaries (SB2) were omitted from our sample, as their combined spectra ensure that an accurate fundamental parameter estimation is impossible as long as their flux ratios are not known. Among the GAUDI sample, we discovered a few candidate spectroscopic binaries, which were not known to be SB2s. It concerns HD 173003 (B5), HD 181474 (B5), HD 42959 (B8), HD 50982 (B8, variable star), HD 51150 (B8, clearly asymmetric profiles), HD 181761 (B8), HD 45953 (B9, variable star), and HD 46165 (B9).

For the few available SARG data, only the default normalized spectra were inserted into the database and we failed to place our hands on the raw data, which would be needed for a careful re-rectification. The poor continuum rectification is particularly clear from $\mathrm{H} \beta$, but also shows up in other line profiles. Moreover, the spectral coverage of the SARG spectra is too small to deduce any useful information. Therefore, we excluded all SARG spectra from our sample. The same accounts for the CATANIA spectra, for which the spectral quality was too poor for a detailed spectral analysis, so these spectra were also omitted. We thus restrict this paper to FEROS and ELODIE spectra, having a resolution of 48000 and 50000 , respectively.

The standard FITS data of the FEROS and ELODIE spectra in the GAUDI database contain information about both the normalized spectrum, resulting from the pipeline reduction, and the unnormalized spectrum. Since the quality of normalization turned out to be insufficient for our detailed analyses, one of us (TM) redid the normalization for all spectral ranges around the diagnostic lines in a uniform way using $\operatorname{IRAF}^{4}$ to ensure a homogeneous treatment of the sample.

Among the GAUDI sample, we find, as expected, a large number of rapid rotators. Their spectra usually contain insufficient line information because of their high projected rotational velocities. Often, only the Balmer lines and the strongest He I lines (i.e. He I 4026, 4471 and 4922) can be detected, while all $\mathrm{Si}$ lines are lost. The stars that were discarded for this reason are HD $182519\left(\mathrm{~B} 5,>300 \mathrm{~km} \mathrm{~s}^{-1}\right)$, HD 50751 (B8, $\left.>200 \mathrm{~km} \mathrm{~s}^{-1}\right)$, HD $56006\left(\mathrm{~B} 8,>150 \mathrm{~km} \mathrm{~s}^{-1}\right)$, HD $45515(\mathrm{~B} 8 \mathrm{~V}$, $\left.>190 \mathrm{~km} \mathrm{~s}^{-1}\right)$, HD $182786\left(\mathrm{~B} 8,>160 \mathrm{~km} \mathrm{~s}^{-1}\right)$, HD 50252 (B9 V, $>140 \mathrm{~km} \mathrm{~s}^{-1}$ ), HD 169225 (B9, >160 $\mathrm{km} \mathrm{s}^{-1}$ ), HD 171931 (B9, $>250 \mathrm{~km} \mathrm{~s}^{-1}$ ), HD $174836\left(\mathrm{~B} 9,>140 \mathrm{~km} \mathrm{~s}^{-1}\right), 176258$ (B9 V, $>160 \mathrm{~km} \mathrm{~s}^{-1}$ ), HD $179124\left(\right.$ B9 V, $\left.>270 \mathrm{~km} \mathrm{~s}^{-1}\right)$, and HD 45760 (B9.5 V, $>190 \mathrm{~km} \mathrm{~s}^{-1}$ ). For some of the GAUDI stars, we were able to fit the spectrum despite the high projected rotational velocity, but their resulting parameters cannot be very reliable. This concerns HD 51507 (B3 V, $148 \mathrm{~km} \mathrm{~s}^{-1}$ ), HD 45418 (B5, $237 \mathrm{~km} \mathrm{~s}^{-1}$ ), HD 46487 (B5 Vn, $265 \mathrm{~km} \mathrm{~s}^{-1}$ ), HD 178744 (B5 $\left.\mathrm{Vn}, 224 \mathrm{~km} \mathrm{~s}^{-1}\right)$, HD $43461\left(\mathrm{~B} 6 \mathrm{~V}, 210 \mathrm{~km} \mathrm{~s}^{-1}\right)$, HD 44720 (B8, $160 \mathrm{~km} \mathrm{~s}^{-1}$ ), HD 49643 (B8 IIIn, $296 \mathrm{~km} \mathrm{~s}^{-1}$ ), HD 173370 (B9V, $282 \mathrm{~km} \mathrm{~s}^{-1}$, double-peaked $\mathrm{H} \alpha$ profile), HD 179124 (B9 $\mathrm{V}, 278 \mathrm{~km} \mathrm{~s}^{-1}$ ), and HD $181690\left(\mathrm{~B} 9 \mathrm{~V}, 189 \mathrm{~km} \mathrm{~s}^{-1}\right)$. Their parameters can be found in Table 3 .

For other targets, we have only spectra of insufficient quality that cannot be used for spectral line-fitting purposes. It concerns HD 48691 (B0.5 IV), HD 168797 (B3 Ve), HD 178129 (B3 Ia), HD 57608 (B8 III, possible instrumental problem), HD 44654 (B9), HD 45257 (B9), and HD 53204 (B9).

For a few stars, we have both an ELODIE and a FEROS spectrum, e.g., for HD 174069. From inspection of the spectra, it is immediately clear that there are differences between the line profiles, the wings of the Balmer lines in particular being far less pronounced in the ELODIE spectra (see Fig. 4). Other stars, for which we have both a FEROS and an ELODIE spectrum available, also show the same discrepancy, so there seems to be a

\footnotetext{
4 IRAF (Image Reduction and Analysis Facility) is distributed by the National Optical Astronomy Observatories, operated by the Association of Universities for Research in Astronomy, Inc., under cooperative agreement with the National Science Foundation, USA.
} 

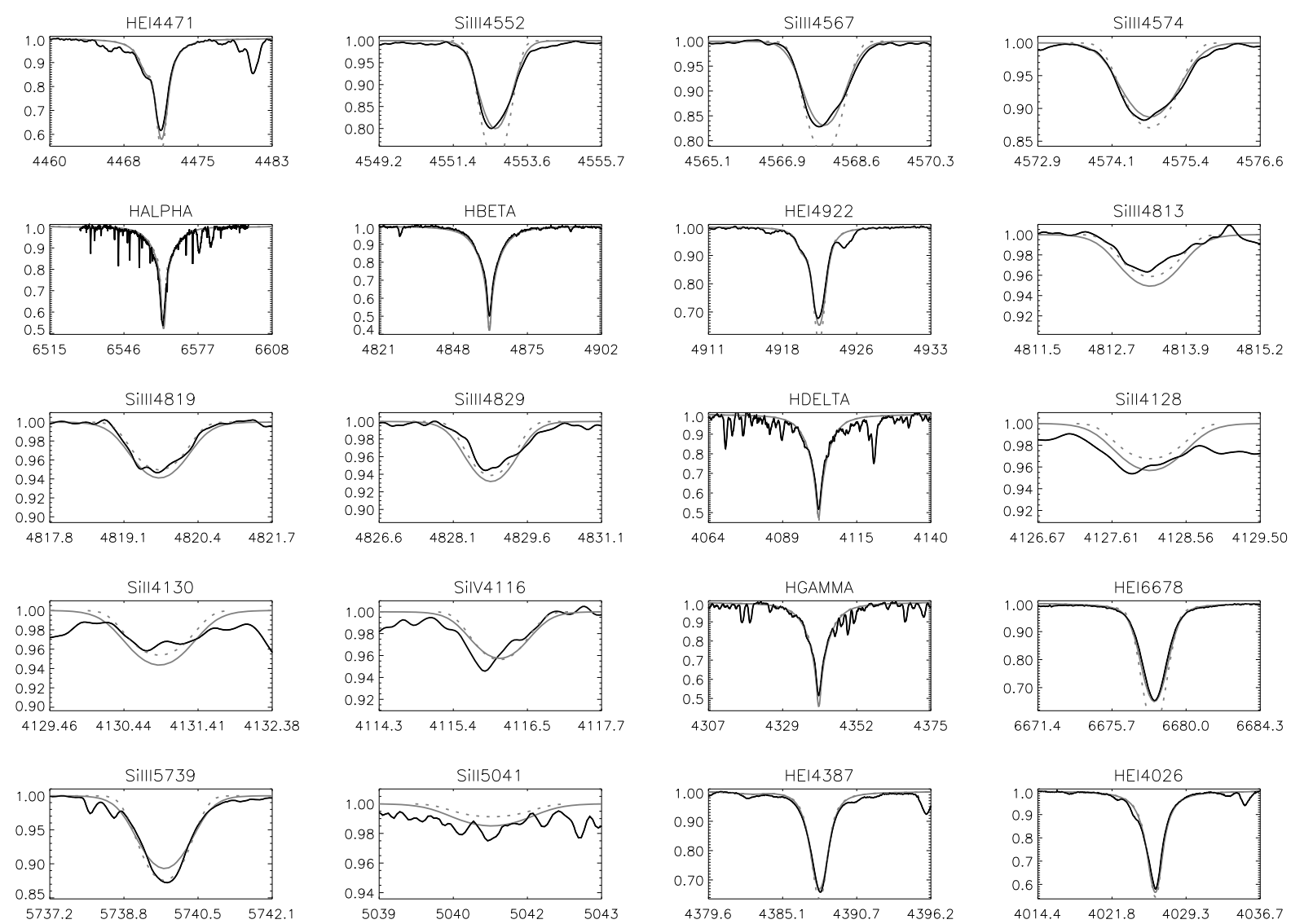

Fig. 3. Illustration of the effects of time-independent broadening on the derived parameters for 12 Lac (B2 III). The observed line profiles (solid black lines) are fitted using AnalyseBstar. Inclusion of time-independent broadening (by means of macroturbulence) yields the solid grey fit, with optimum values of $v \sin i=44 \mathrm{~km} \mathrm{~s}^{-1}$ and $v_{\text {macro }}=37 \mathrm{~km} \mathrm{~s}^{-1}$. Forcing the macroturbulence to be zero leaves all parameters but the Si abundance and the microturbulence unaltered. The resulting fit is represented by the grey dashed lines.

systematic effect. We fitted both spectra and derived a different set of parameters. The difference in $\log g$ is large $(>0.5 \mathrm{dex})$ and certainly worrisome. To investigate this problem, we searched for spectra in the FEROS and ELODIE archives and realized that the coaddition of the ELODIE spectra by the pipeline is far less accurate than for the FEROS spectra. This is because of an inaccurate correction for the blaze function by the ELODIE pipeline. Due to this, we also repeated the merging of the orders for the ELODIE spectra, before the normalization. This led to a much closer agreement with the available FEROS spectra. We thus advise future users against working with the merged and normalized spectra from GAUDI, but rather to return to the original spectra in the FEROS and ELODIE archives, and not only redo the normalization but even the merging in the case of the ELODIE spectra. Unfortunately, not all stars had a spectrum available in the ELODIE archives though, e.g., for HD 47887 (B2 III), HD 52559 (B2 IV-V), HD 48977 (B2.5 V), HD 50228 (B5), HD 57291 (B5), HD 51892 (B7 III), HD 52206 (B8), and HD 53202 (B9), we could not find the original spectrum, so we removed these stars from our analysis. For HD 43317 (B3 IV), the archival spectrum was not usable.

Finally, for 17 of the remaining stars, we could not find a satisfactory fit, and we decided to leave them out of the sample. One possible explanation might be that they are single-lined binaries (see also Massey et al. 2009 who encountered similar problems when analyzing a large sample of LMC/SMC O-stars).

Altogether, this, unfortunately, reduces our sample dramatically, and we are left only with a little more than a third of the initial targets in the database: 66 out of 187 objects. Moreover, most of the objects are late B-type stars. Figure 5 shows a histogram of the breakdown of the sample in terms of spectral subtype. The ratio given above each bin indicates the number of analyzed targets to the total amount available before the selection procedure.

\subsection{Analysis results}

\subsubsection{Example fits}

To illustrate the obtained quality of the final fits, we show in Figs. 6 to 8 some examples of the resulting spectral fits for three very different stars included in GAUDI: a "cool" giant, a hot supergiant, and a middle type dwarf. The corresponding physical parameters are listed in Table 3, which summarizes the derived stellar parameters of the analysed GAUDI B type stars ${ }^{5}$.

\subsubsection{Comments on individual stars}

In what follows, we discuss a few of the sample stars individually, because of certain particularities.

\footnotetext{
5 All other spectral line fits, and their corresponding parameters, can be found at http://www. ster.kuleuven.be/ karolien/ AnalyseBstar/Bstars/
} 
Table 3. Stellar parameters of the analyzed GAUDI B type stars as derived from the best-fit model to the observations found by AnalyseBstar.

\begin{tabular}{|c|c|c|c|c|c|c|c|c|c|c|c|}
\hline $\begin{array}{r}\text { HD } \\
\text { number }\end{array}$ & $\begin{array}{l}\text { SpT } \\
\text { SIMBAD }\end{array}$ & $\begin{array}{l}T_{\mathrm{eff}^{a}} \\
(\mathrm{~K})\end{array}$ & $\begin{array}{l}\log g_{c} \\
(\mathrm{cgs})\end{array}$ & $\frac{n(\mathrm{He})}{n(\mathrm{H})} b$ & $\log \frac{n(\mathrm{Si})}{n(\mathrm{H})} b$ & $\begin{array}{r}\xi^{b} \\
\left(\mathrm{~km} \mathrm{~s}^{-1}\right)\end{array}$ & $\begin{array}{c}v \sin i \\
\left(\mathrm{~km} \mathrm{~s}^{-1}\right)\end{array}$ & $\begin{array}{r}v_{\text {macro }} \\
\left(\mathrm{km} \mathrm{s}^{-1}\right)\end{array}$ & $\begin{array}{c}\text { Suspected } \\
\text { pulsator }\end{array}$ & Flag $^{c}$ & Remarks \\
\hline 48434 & B0III & 28000 & $3.11 \pm 0.10$ & 0.10 & -4.44 & 15 & $62 \pm 3$ & 31 & yes & 4 & $\begin{array}{l}\text { ELODIE, poor quality spectrum, } \\
\text { He lines too strong, Balmer } \\
\text { lines not well fitted } \\
\text { due to bumps in blue wings }\end{array}$ \\
\hline 52382 & $\mathrm{~B} 1 \mathrm{Ib}$ & 23000 & $2.71 \pm 0.10$ & 0.15 & -4.67 & 20 & $56 \pm 5$ & 53 & yes & 4 & $\begin{array}{l}\text { P Cygni profile } \mathrm{H} \alpha \text { not reproduced, } \\
\mathrm{H} \beta \text { and } \mathrm{H} \gamma \text { not well fitted }\end{array}$ \\
\hline 170580 & $\mathrm{~B} 2 \mathrm{~V}$ & 20000 & $4.10 \pm 0.10$ & 0.10 & -4.81 & 6 & $11 \pm 1$ & 0 & no & 1 & $N(\mathrm{He}) / N(\mathrm{H})$ lower than solar \\
\hline 44700 & B3 V & 17500 & $3.80 \pm 0.10$ & 0.10 & -4.93 & 6 & $8 \pm 1$ & 0 & no & 1 & $N(\mathrm{He}) / N(\mathrm{H})$ lower than solar \\
\hline 181074 & B3 & 20000 & $3.64 \pm 0.10$ & 0.10 & -4.35 & 12 & $133 \pm 7$ & 0 & no & 2 & probably B2, fast rotator \\
\hline 45418 & B5 & 16000 & $4.0 \pm 0.20$ & 0.10 & -4.49 & 3 & $237 \pm 28$ & 0 & no & 3 & \\
\hline 46487 & $\mathrm{~B} 5 \mathrm{Vn}$ & 14500 & $3.69 \pm 0.10$ & 0.15 & -4.49 & 3 & $265 \pm 5$ & 0 & no & 3 & \\
\hline 48215 & B5V & 14500 & $3.82 \pm 0.10$ & 0.10 & -4.49 & 3 & $98 \pm 3$ & 48 & yes & 1 & only Si II \\
\hline 54596 & B5 & 20000 & $3.31 \pm 0.05$ & 0.10 & -4.48 & 6 & $69 \pm 6$ & 53 & yes & 2 & only Si III, probably B2 \\
\hline 58973 & B5 & 15000 & $3.43 \pm 0.20$ & 0.10 & -4.49 & 3 & $94 \pm 1$ & 50 & yes & 1 & \\
\hline 177880 & $\mathrm{~B} 5 \mathrm{~V}$ & 14500 & $3.81 \pm 0.10$ & 0.10 & -4.49 & 3 & $49 \pm 1$ & 23 & yes & 1 & \\
\hline 178744 & $\mathrm{~B} 5 \mathrm{Vn}$ & 14500 & $3.71 \pm 0.20$ & 0.10 & -4.49 & 12 & $224 \pm 5$ & 0 & no & 3 & \\
\hline 43461 & $\mathrm{~B} 6 \mathrm{~V}$ & 13500 & $3.35 \pm 0.10$ & 0.10 & -4.79 & 3 & $210 \pm 5$ & 0 & no & 3 & \\
\hline 48807 & B7Iab & 12500 & $2.00 \pm 0.10$ & 0.10 & -4.28 & 6 & $24 \pm 1$ & 27 & yes & 1 & \\
\hline 51360 & B7III & 13500 & $3.12 \pm 0.10$ & 0.10 & -3.93 & 3 & $73 \pm 1$ & 55 & yes & 1 & very high $\mathrm{Si}$ abundance \\
\hline 42677 & B8 & 11000 & $3.55 \pm 0.20$ & 0.10 & -4.79 & 6 & $121 \pm 14$ & 104 & yes & 2 & probably B9 \\
\hline 44720 & $\mathrm{~B} 8$ & 14000 & $3.96 \pm 0.10$ & 0.10 & -4.19 & 3 & $160 \pm 5$ & 0 & no & 2 & probably $\mathrm{B} 5$ or B6 \\
\hline 45153 & B8 & 11000 & $3.66 \pm 0.10$ & 0.10 & -4.49 & 3 & $142 \pm 4$ & 0 & no & 2 & probably B9 \\
\hline 45284 & B8 & 15000 & $4.10 \pm 0.10$ & 0.10 & -4.19 & 3 & $52 \pm 5$ & 42 & yes & 2 & $\begin{array}{l}\text { SPB with magnetic field, skew Si II lines, } \\
\text { probably B5 }\end{array}$ \\
\hline 45397 & B8 & 11500 & $3.59 \pm 0.10$ & 0.20 & -4.79 & 6 & $156 \pm 12$ & 0 & no & 3 & \\
\hline 45515 & B8V & 11000 & $4.18 \pm 0.10$ & 0.15 & -4.49 & 3 & $190 \pm 5$ & 0 & no & 2,3 & probably B9 \\
\hline 46616 & B8 & 17500 & $4.20 \pm 0.50$ & 0.10 & -4.24 & 3 & $8 \pm 1$ & 7 & yes & 5 & He-weak star \\
\hline 47964 & B8III & 11500 & $3.01 \pm 0.20$ & 0.10 & -4.79 & 3 & $49 \pm 1$ & 44 & yes & 1 & \\
\hline 48497 & B8 & 14000 & $3.70 \pm 0.10$ & 0.10 & -4.58 & 3 & $14 \pm 1$ & 13 & yes & 4,6 & $N(\mathrm{He}) / N(\mathrm{H})$ lower than solar \\
\hline 49481 & B8 & 11000 & $2.70 \pm 0.10$ & 0.20 & -4.79 & 3 & $9 \pm 1$ & 13 & yes & 2,6 & probably B9 \\
\hline 49643 & B8IIIn & 14500 & $3.88 \pm 0.10$ & 0.10 & -4.19 & 3 & $296 \pm 2$ & 13 & no & 3 & \\
\hline 49886 & B8 & 10000 & $3.30 \pm 0.20$ & 0.10 & -4.79 & 6 & $8 \pm 1$ & 16 & yes & 4,6 & \\
\hline 49935 & B8 & 12500 & $3.33 \pm 0.20$ & 0.10 & -4.79 & 6 & $92 \pm 9$ & 72 & yes & 1 & $\log g$ may be too low? \\
\hline 50251 & B8V & 11500 & $3.00 \pm 0.20$ & 0.10 & -4.79 & 3 & $9 \pm 1$ & 17 & yes & 1 & $\begin{array}{l}\text { emission line } \\
\text { around Si III } 4813 \text { and Si IV } 4212\end{array}$ \\
\hline 50513 & B8 & 11500 & $4.04 \pm 0.10$ & 0.10 & -4.79 & 6 & $112 \pm 5$ & 80 & yes & 1 & \\
\hline 55793 & B8 & 11000 & $3.05 \pm 0.05$ & 0.20 & -4.79 & 3 & $101 \pm 1$ & 56 & yes & 2 & probably $\mathrm{B} 9, \log g$ may be too low? \\
\hline 56446 & B8III & 11500 & $3.21 \pm 0.20$ & 0.20 & -4.19 & 3 & $229 \pm 32$ & 13 & no & 3 & \\
\hline 170795 & B8 & 15500 & $4.01 \pm 0.10$ & 0.10 & -4.49 & 3 & $79 \pm 7$ & 37 & yes & 2 & probably B5 \\
\hline 171247 & B8IIIsp & 10000 & $2.83 \pm 0.10$ & 0.10 & -4.19 & 6 & $66 \pm 2$ & 20 & yes & 4 & peculiar line behaviour \\
\hline 173673 & B8 & 10000 & $2.90 \pm 0.10$ & 0.20 & -4.49 & 3 & $25 \pm 2$ & 26 & yes & 4,6 & too low $\log g$ ? \\
\hline 179761 & B8II-III & 12500 & $3.30 \pm 0.05$ & 0.10 & -4.49 & 3 & $17 \pm 1$ & 12 & yes & 1 & \\
\hline 180760 & B8 & 17000 & $4.05 \pm 0.10$ & 0.10 & -4.49 & 6 & $167 \pm 3$ & 0 & no & 3 & wrong spectral type? \\
\hline 44321 & B9 & 11000 & $3.62 \pm 0.05$ & 0.10 & -4.79 & 6 & $90 \pm 3$ & 30 & yes & 1 & \\
\hline 44354 & B9 & 13000 & $3.94 \pm 0.05$ & 0.15 & -4.79 & 10 & $121 \pm 5$ & 72 & yes & 2 & probably B7 or B8 \\
\hline 45050 & B9V & 11500 & $3.86 \pm 0.10$ & 0.10 & -4.79 & 10 & $140 \pm 47$ & 0 & no & 3 & \\
\hline 45516 & B9 & 13000 & $3.90 \pm 0.10$ & 0.10 & -4.49 & 6 & $281 \pm 55$ & 12 & no & 3 & \\
\hline 45657 & B9 & 11000 & $4.24 \pm 0.10$ & 0.10 & -4.79 & 3 & $153 \pm 36$ & 0 & no & 3 & \\
\hline 45709 & B9 & 11000 & $3.95 \pm 0.30$ & 0.20 & -4.79 & 10 & $231 \pm 24$ & 10 & no & 3 & \\
\hline 45975 & B9 & 11000 & $3.51 \pm 0.10$ & 0.15 & -4.79 & 3 & $54 \pm 1$ & 56 & yes & 1 & $v_{\text {macro }}$ too high \\
\hline 46138 & B9 & 12000 & $3.93 \pm 0.05$ & 0.10 & -4.19 & 3 & $100 \pm 5$ & 0 & no & 4 & \\
\hline 46886 & B9 & 11000 & $3.00 \pm 0.10$ & 0.10 & -4.79 & 3 & $16 \pm 3$ & 23 & yes & 1 & $\log g$ too low? \\
\hline 47278 & B9 & 10000 & $3.60 \pm 0.05$ & 0.10 & -4.79 & 3 & $31 \pm 4$ & 25 & yes & 2 & rather $\mathrm{A} 0$ \\
\hline 48808 & B9 & 12000 & $3.21 \pm 0.10$ & 0.10 & -79 & 3 & $47 \pm 14$ & 63 & yes & 1 & $v_{\text {macro }}$ too high, rather $\mathrm{B} 8$ \\
\hline 48957 & B9 & 12000 & $3.10 \pm 0.10$ & 0.10 & -4.79 & 3 & $24 \pm 2$ & 23 & yes & 4 & $T_{\text {eff }}$ too low?, wrong spectral type? \\
\hline 49123 & B9 & 10000 & $3.41 \pm 0.05$ & 0.10 & -4.79 & 3 & $45 \pm 2$ & 0 & no & 2 & rather $\mathrm{A} 0$ \\
\hline 52312 & B9III & 11000 & $3.04 \pm 0.05$ & 0.20 & -4.49 & 3 & $175 \pm 5$ & 0 & no & 3 & \\
\hline 53004 & B9 & 11000 & $3.91 \pm 0.20$ & 0.10 & -4.79 & 6 & $51 \pm 7$ & 54 & yes & 1 & \\
\hline 54761 & B9 & 11500 & $3.11 \pm 0.10$ & 0.10 & -4.79 & 6 & $53 \pm 1$ & 71 & yes & 1 & $\begin{array}{l}\text { Balmer line cores slightly } \\
\text { refilled, } \log g \text { too high? }\end{array}$ \\
\hline 54929 & B9 & 10000 & $3.11 \pm 0.10$ & 0.10 & -4.49 & 3 & $46 \pm 1$ & 107 & yes & 4 & $\begin{array}{l}\text { fast rotator, difficult to fit, } \\
\text { too high } v_{\text {macro }}\end{array}$ \\
\hline 56613 & B9 & 13000 & $3.92 \pm 0.20$ & 0.10 & -4.79 & 6 & $91 \pm 7$ & 36 & yes & 2 & rather, B7 or B8 \\
\hline
\end{tabular}


Table 3. continued.

\begin{tabular}{|c|c|c|c|c|c|c|c|c|c|c|}
\hline $\begin{array}{cl}\text { HD } & \text { SpT } \\
\text { number } & \text { SIMBAD }\end{array}$ & $\begin{array}{l}T_{\text {eff }}^{a} \\
(\mathrm{~K})\end{array}$ & $\begin{array}{l}\log g_{c} \\
(\mathrm{cgs})\end{array}$ & $\frac{n(\mathrm{He})}{n(\mathrm{H})} b$ & $\log \frac{n(\mathrm{Si})}{n(\mathrm{H})} b$ & $\begin{array}{r}\xi^{b} \\
\left(\mathrm{~km} \mathrm{~s}^{-1}\right)\end{array}$ & $\begin{array}{c}v \sin i \\
\left(\mathrm{~km} \mathrm{~s}^{-1}\right)\end{array}$ & $\begin{array}{r}v_{\text {macro }} \\
\left(\mathrm{km} \mathrm{s}^{-1}\right)\end{array}$ & $\begin{array}{l}\text { Suspected } \\
\text { pulsator }\end{array}$ & Flag $^{c}$ & Remarks \\
\hline 172850 B9 & 11000 & $3.62 \pm 0.10$ & 0.10 & -4.49 & 3 & $77 \pm 4$ & 0 & no & 1 & \\
\hline $173370 \mathrm{~B} 9 \mathrm{~V}$ & 11500 & $3.46 \pm 0.10$ & 0.10 & -4.79 & 15 & $282 \pm 8$ & 0 & no & 4 & $\begin{array}{l}\text { very fast rotator, no } \mathrm{Si} \text { lines available, } \\
\text { double-peaked } \mathrm{H} \alpha \text { profile, } \\
\mathrm{H} \beta \text { refilled on both sides of the line core }\end{array}$ \\
\hline 173693 B9 & 10000 & $3.32 \pm 0.10$ & 0.10 & -4.79 & 3 & $60 \pm 1$ & 22 & yes & 2 & rather A0 \\
\hline 174701 B9 & 12500 & $3.68 \pm 0.10$ & 0.15 & -4.49 & 3 & $170 \pm 2$ & 10 & no & 3 & \\
\hline 175640 B9III & 10000 & $3.20 \pm 0.10$ & 0.15 & -4.49 & 3 & $7 \pm 1$ & 10 & yes & 2 & rather $\mathrm{A} 0$ \\
\hline 176076 B9 & 10000 & $3.41 \pm 0.10$ & 0.10 & -4.79 & 3 & $42 \pm 2$ & 0 & no & 2 & rather $\mathrm{A} 0$ \\
\hline 176158 B9 & 13500 & $3.64 \pm 0.10$ & 0.15 & -4.79 & 3 & $121 \pm 11$ & 0 & no & 2 & rather B8 \\
\hline 179124 B9V & 12000 & $3.45 \pm 0.10$ & 0.10 & -4.49 & 15 & $277 \pm 6$ & 0 & no & 3 & \\
\hline 181440 B9III & 11500 & $3.61 \pm 0.50$ & 0.10 & -4.49 & 3 & $55 \pm 7$ & 32 & yes & 1 & \\
\hline $181690 \mathrm{~B} 9 \mathrm{~V}$ & 13000 & $3.42 \pm 0.10$ & 0.10 & -4.49 & 6 & $189 \pm 5$ & 0 & no & 3 & \\
\hline 182198 B9V & 11000 & $3.10 \pm 0.10$ & 0.10 & -4.79 & 3 & $23 \pm 1$ & 12 & yes & 1 & \\
\hline
\end{tabular}

Notes. In some cases, we need an additional broadening to explain the line profiles. Stars suspected to be pulsators on the basis of their significant $v_{\text {macro }}$ are indicated. ${ }^{(a)}$ The formal uncertainty in the effective temperature is $1000 \mathrm{~K}$ for $T_{\text {eff }}>20,000 \mathrm{~K}$ and $T_{\text {eff }}<15000 K$, and $500 \mathrm{~K}$ for $T_{\text {eff }}<15,000 K \leq T_{\text {eff }} \leq 20000 K{ }^{(b)}$ The formal uncertainty in the microturbulence and the He and Si abundance is half of the grid step in most cases: $2 \mathrm{~km} \mathrm{~s}^{-1}$ and $0.025 \mathrm{dex}$, respectively. For objects below $15000 \mathrm{~K}$, we adopt somewhat larger errors (3- $\sigma$ deviation corresponding to 2 grid steps): $3 \mathrm{~km} \mathrm{~s}^{-1}, 0.033$ and 0.2 dex, respectively. ${ }^{(c)}$ We attribute a flag to each star, to represent the obtained fit quality or problems we encountered during the fitting procedure: 1 - well-fitted, 2 - probably a wrong spectral type (about $1000 \mathrm{~K}$ or more away from the calibrations, proposed spectral type indicated in the "remarks"-column), 3 - no or hardly any Si lines available, mostly due to rapid rotation, 4 - not very reliable fit (for other reasons than fast rotation, see "remarks"), 5 - He weak/Si strong, 6 - fitting of $\mathrm{H} \alpha, \mathrm{H} \beta$, and $\mathrm{H} \gamma$ line profiles complicated due to very broad wings that abruptly change into very narrow line cores ( $\log g$ may be less accurate).

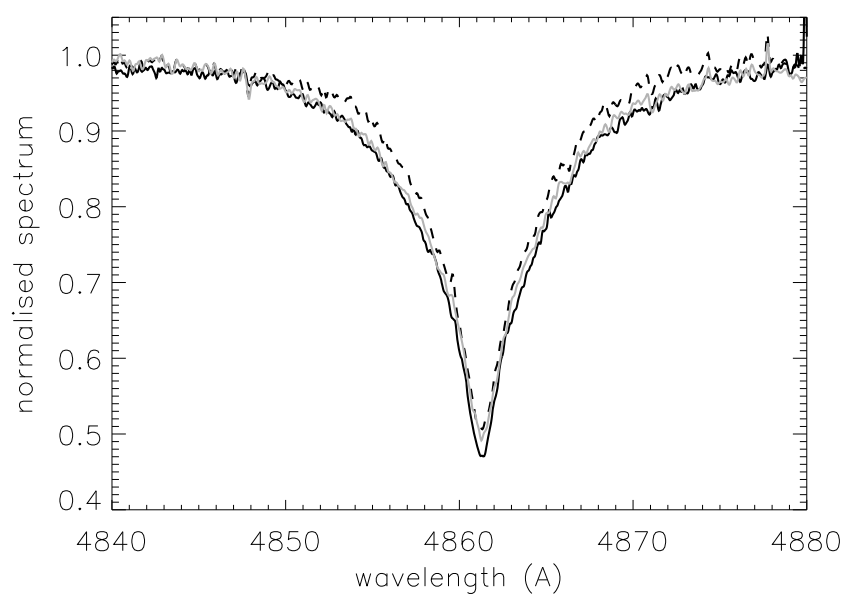

Fig. 4. HD 174069: Significant (and apparently systematic) discrepancies are observed between the line profiles of the FEROS (solid black line) and the ELODIE (dashed black line) spectrum from the GAUDI database. This leads to considerable discrepancies in the derived stellar parameters. In grey, we show the normalized ELODIE spectrum obtained from the ELODIE archives, which shows a much better agreement with the available FEROS spectra.

HD 45284. The derived effective temperature is in complete agreement with what was found by Hubrig et al. (2006). This star is an SPB exhibiting a magnetic field. The combination of effective temperature and gravity derived from Geneva photometry (Hubrig et al. 2006) infers that the star is just outside the main sequence towards the high-gravity side. With our newly derived $\log g$, it now falls within the expected instability domain of the SPB stars.

HD 46616 (B8). Is a clear example of a He-weak star: all He I-lines have extremely low equivalent widths compared to "normal" B stars. Even though the hydrogen and Si lines are

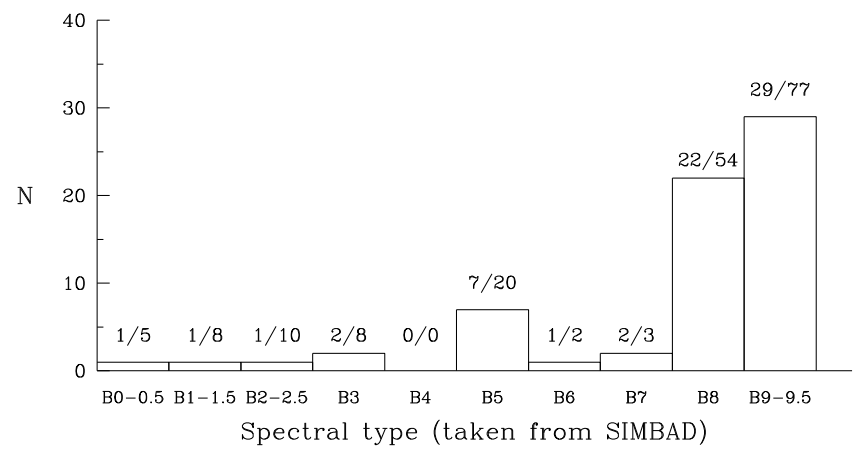

Fig. 5. Histogram of the analyzed stars within the GAUDI B star sample. The ratio marked above each bin indicates the number of analyzed targets compared to the total amount that was available before the selection procedure, e.g., 1/5 means that there were 5 objects with this spectral type in the database, but that, for certain reasons, we were only able to analyze 1 of them. In total, 66 out of 187 objects could be analyzed.

reproduced well, the He lines are predicted to be too strong in our grid models ${ }^{6}$.

HD 48106 (B8). Is oxygen poor based on the non-detection of the O I 7771-7775 triplet and is either He weak or Si strong. We were unable to fit both the $\mathrm{He}$ and $\mathrm{Si}$ lines at the same time.

For HD 46340 (B8). Only He I 4471 and Si II 5041-5056 are visible. This was only sufficient to make a very rough estimate of the fundamental parameters: $T_{\mathrm{eff}} \approx 10000 \mathrm{~K}, \log g \approx 4.1$, negligible wind, and solar He abundance.

On the hot side of the B-type domain, the analysis of the early-type stars (e.g., HD 172488 (B0.5 V), HD 52918 (B1 V),

\footnotetext{
6 The lowest considered He-abundance in our grid is $n(\mathrm{He}) / n(\mathrm{H})=0.1$, see Sect. B.8.
} 

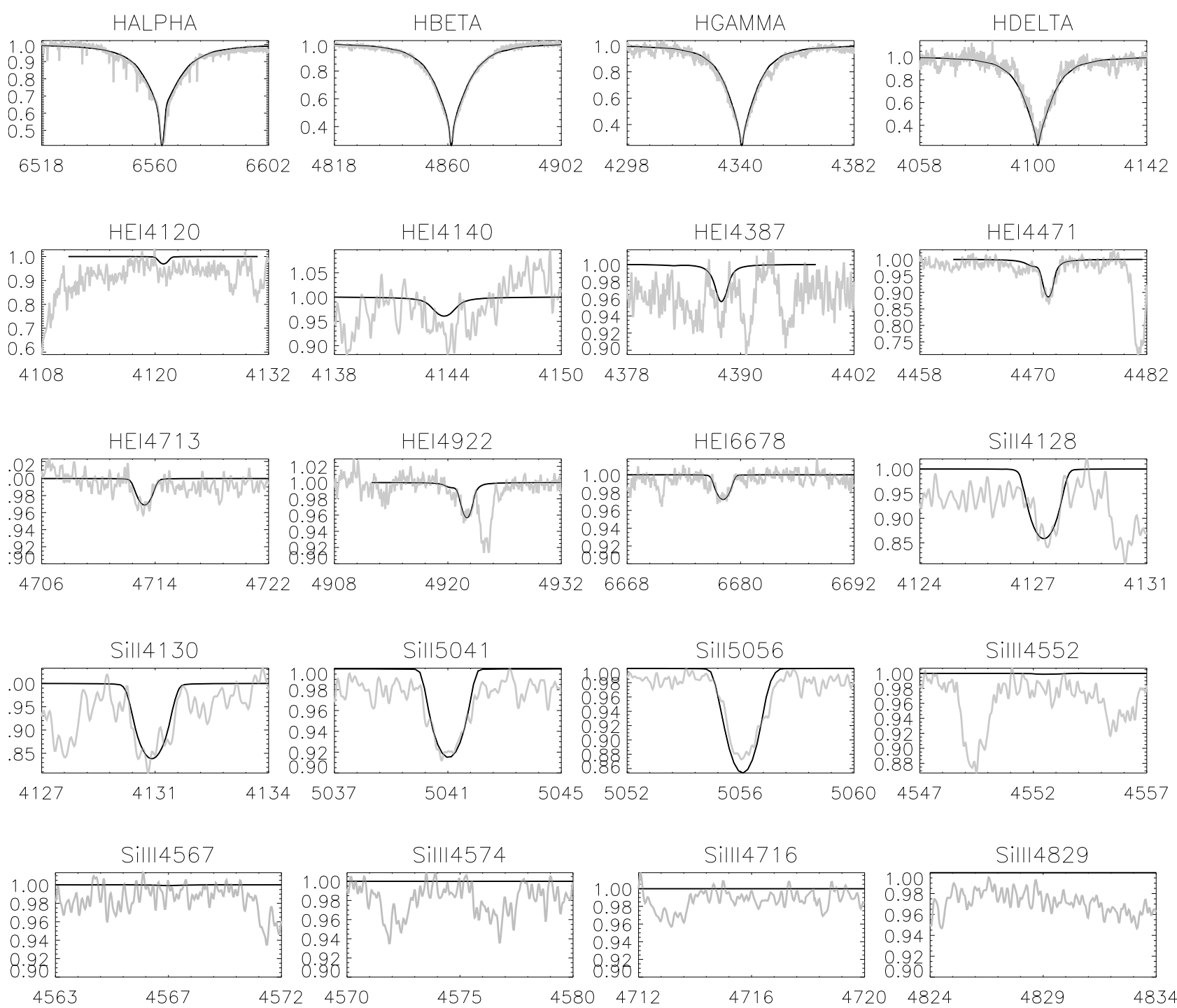

Fig. 6. Example of the line profile fits for a "cool" giant which has, meanwhile, been observed by CoRoT (Miglio et al., in preparation): HD 181440 (B9 III). Despite the low quality of the data, and the fact that we have no more Si III left in this temperature region, we are still able to obtain a satisfying solution.

and HD 173198 (B1 V)) show similar difficulties. The iterative procedure tends to yield too low temperatures because of the lower weight of the Si IV and He II lines as we have only one weak line. Since only Si III is reliable and He I is not affected much by the effective temperature in this range, we are unable to derive trustworthy results.

HD 173370 (B9 V). Shows a double-peaked H $\alpha$ profile. This morphology is typical of a very rapid rotator (in this case, $v \sin i \approx 280 \mathrm{~km} \mathrm{~s}^{-1}$ ), seen almost equator-on. The disk-like feature also strongly affects the $\mathrm{H} \beta$ and $\mathrm{H} \gamma$ profiles.

We also found a few chemically peculiar stars among the B stars in the GAUDI database. They are HD 44948 (B8 Vp), HD 45583 (B8), HD 46616 (B8), and HD 44907 (B9). They have a very rich spectrum, with a forest of sharp spectral lines, complicating the continuum determination and the fitting process, since almost all lines are blended. The He lines of HD 45583 are extremely weak and those of HD 46616 even completely vanish. Following Landstreet (2007), HD 45583 is a periodically variable Ap star. The line spectrum is completely distorted, hence too complicated to fit. The Si II lines are very strong, which indeed points towards a B8 or even B9 star.

\section{Statistical properties of the sample and physical interpretation}

\subsection{Effective temperature scale}

To derive a reliable calibration of the effective temperature as a function of the spectral subclass for B dwarfs, we need enough stars for which we have accurate information about both parameters. Unfortunately, this prerequisite is not fulfilled for our sample, as can be seen from the very large scatter around the existing

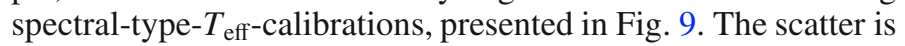
caused by uncertain effective temperatures for some targets and incorrect spectral type designation for others. Indeed, for several stars, we encountered problems in the analysis, primarily because of the absence of Si lines for many rapid-rotating late B type stars. These stars are too cool to have Si III present in their line spectrum, and the Si II lines are completely smooth, so that they become hardly detectable. Even when they remain 
K. Lefever et al.: Fundamental parameters of B stars in the FoV of CoRoT
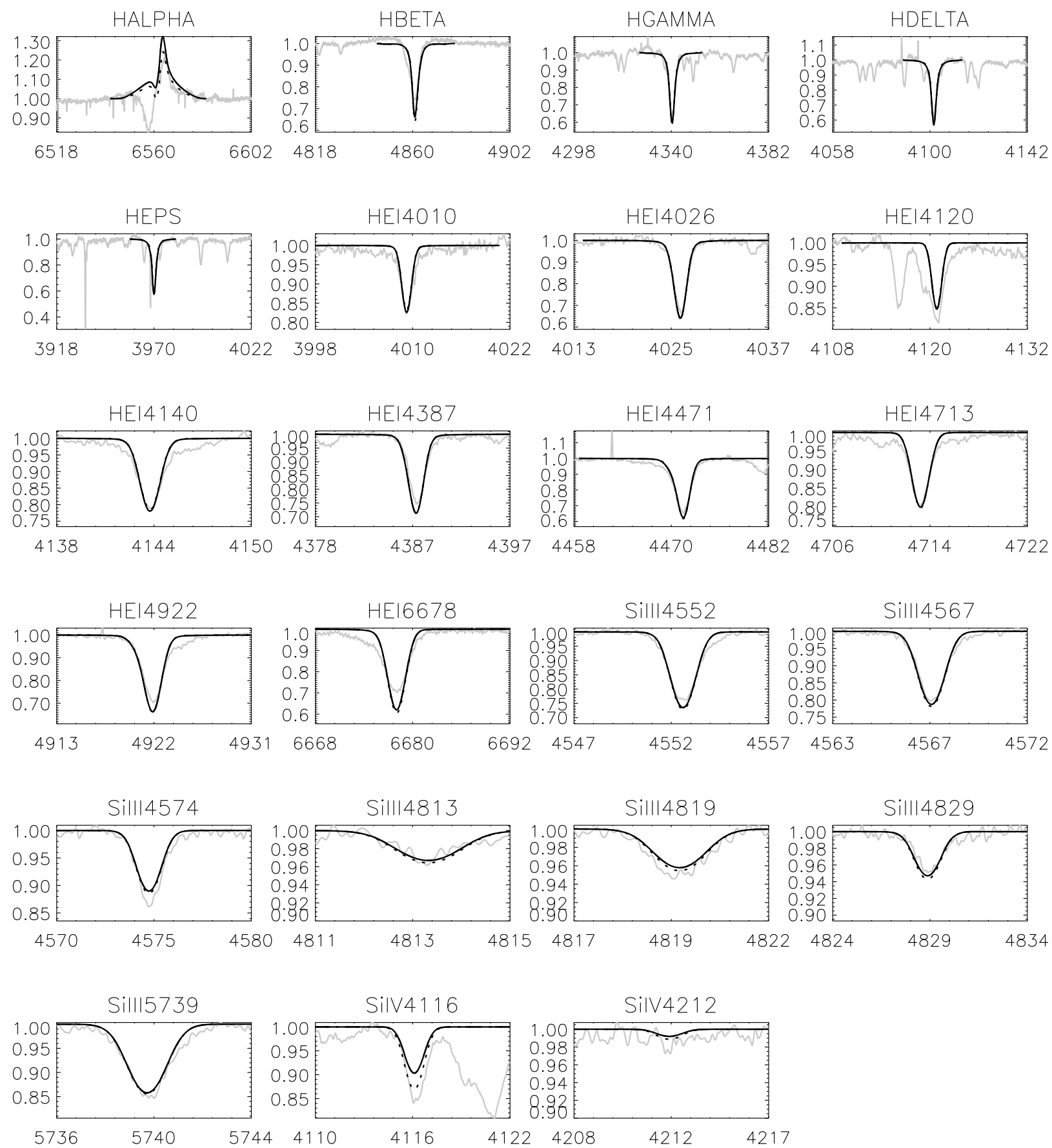

Fig. 7. Example of the line profile fits for the only hot supergiant in our sample: HD 52382 (B1Ib). While the peak of H $\alpha$ is reasonably well reproduced, the blueward absorption trough cannot be fitted at the available grid combinations of wind strength parameter and wind velocity field exponent. Note that no interpolation is performed for these quantities, and that the wind is considered as unclumped, which might explain the obvious mismatch (e.g., Puls et al. 2006, in particular their Fig. 7). The Balmer lines show bumps in the wings. It is not clear whether this is real and due to the strong wind, or if this is a spectral artefact. It also arises in the only cool supergiant in our sample. The interpolated Si abundance $(\log n(\mathrm{Si}) / n(\mathrm{H})=-4.67)$ is higher than the closest grid abundance $(\log n(\mathrm{Si}) / n(\mathrm{H})=-4.79$, plotted here), which explains the observed discrepancies in the Si line cores. By dotted lines, we show the line profiles as they would appear with the interpolated parameters.

visible, the two lines of the Si II 4128-4130 doublet are so heavily blended that they cannot be used any longer. In these cases, the He I lines are the only lines that can be used to estimate the effective temperature, which are, therefore, not reliable enough to use in the derivation of an accurate temperature calibration. On the other hand, several stars for which we were able to derive accurate values of the effective temperature were found to have completely wrong spectral types. We caution against the use of spectral types as they are quite often derived from lowresolution and low-quality spectra. Flags and remarks about the fit quality of individual targets were included in Table 3.

\subsection{The stars in the $\left(T_{\mathrm{eff}}, \log g_{c}\right)$-diagram}

Figure 10 shows the $\left(T_{\text {eff }}, \log g_{c}\right)$-diagram of the analyzed GAUDI stars, along with the position of instability strips. 

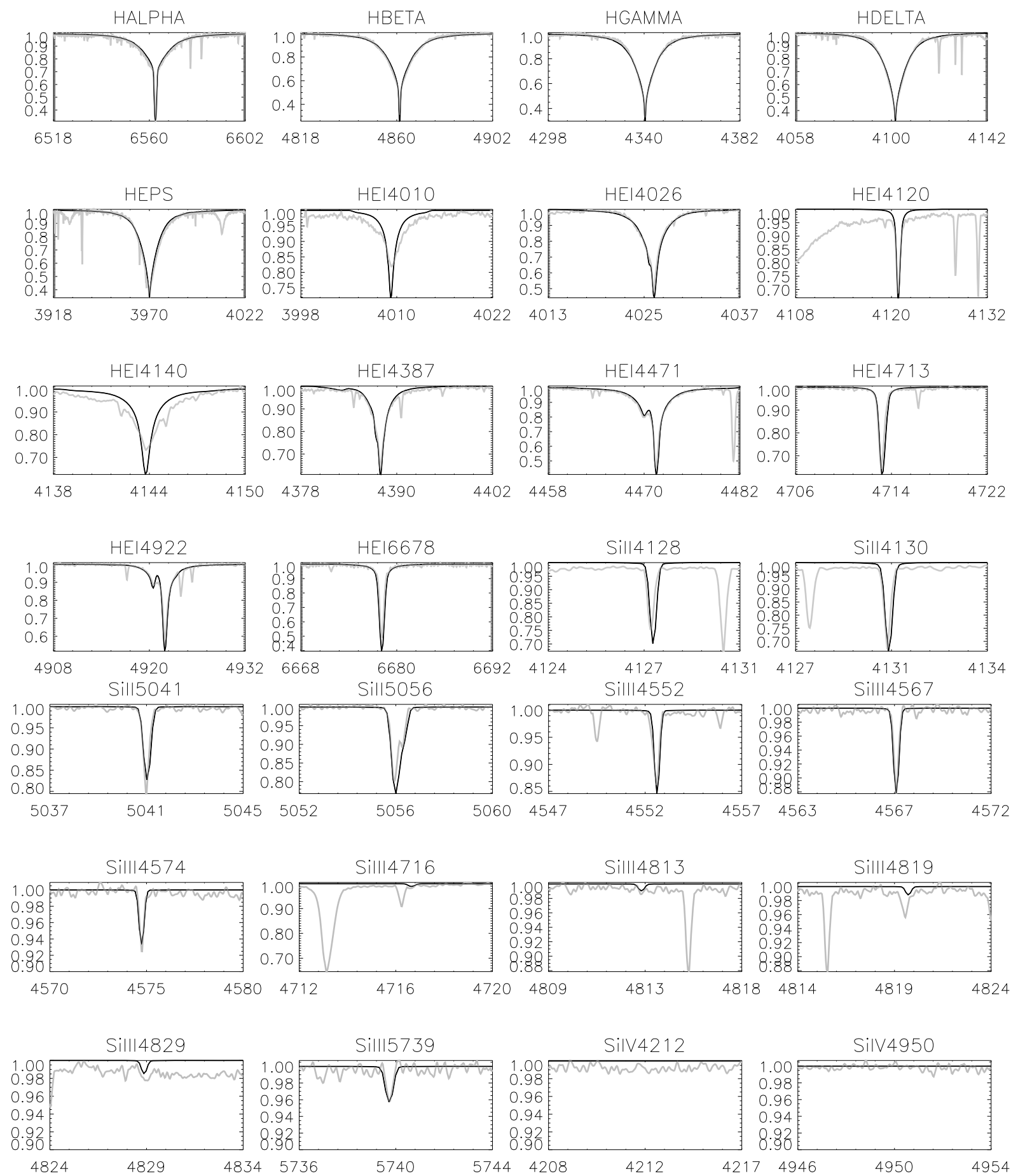

Fig. 8. Example of the line profile fits for an intermediate hot dwarf: HD 44700 (B3 V). The strong lines on the blue side of Si III 4716 and $\mathrm{Si}$ III 4819 are easily mistaken for the Si lines themselves. This is the reason why we always indicate the exact position of the transition during the full preparation process. The poor fits of He I 4010 and 4140 are caused by the incomplete broadening functions of these lines. These lines were not used during the fitting procedure, but only as a double check afterwards (see Appendix B.1). We observe a similar behavior in several other stars. All other lines, even the weakest, fit very well. The shape of He I 4471 is even perfect.

The latter were computed with the evolutionary code CLÉS (Scuflaire et al. 2008) and the pulsation code MAD (Dupret et al. 2002), respectively. These strips were computed for a coreovershoot parameter of 0.2 times the local pressure scale height (Miglio et al. 2007a,b). From seismic modeling of B type stars, it was indeed found that core overshooting occurs, on average, for this value (Aerts 2008). We thus compare the position of the GAUDI stars with the latest models tuned by asteroseismology.
For $40 \%$ of the GAUDI stars (26 out of 66 ), we have information about the luminosity class. All but one (i.e. 9 out of 10) of known luminosity class II or III are indeed situated beyond the terminal age main sequence (TAMS hereafter), so we confirm them to be giants. The only exception is HD 49643, a star of spectral type B8 IIIn, i.e., with spectroscopic lines that may originate from nebulosity. The star is a rapid rotator with a $v \sin i$ of almost $300 \mathrm{~km} \mathrm{~s}^{-1}$. The absence of Si lines because of the high rotation implies that its estimates of $T_{\text {eff }}$ and $\log g$ are less 


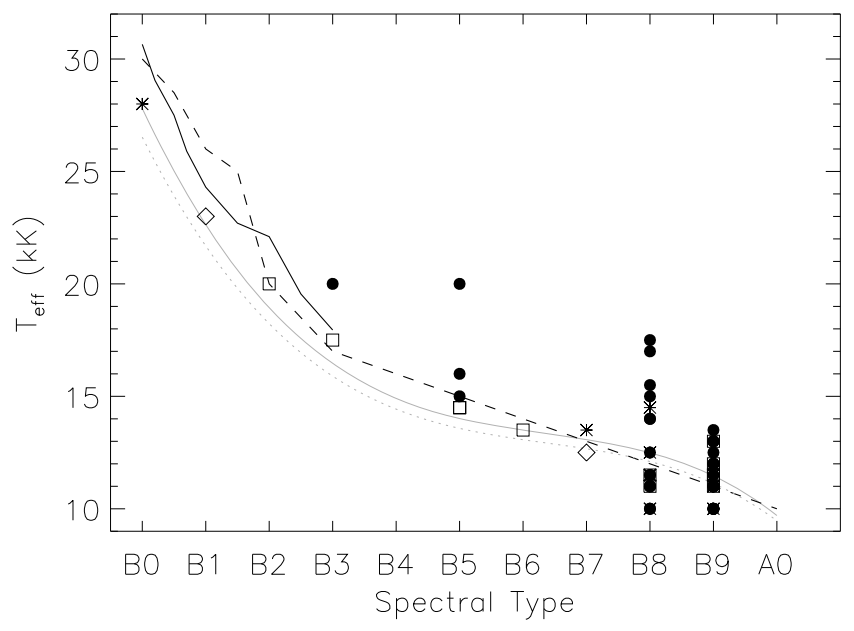

Fig. 9. Effective temperature as a function of spectral type. We compare the position of our sample stars to existing temperature scales: for Galactic dwarfs, for spectral types up to B3 (Trundle et al. 2007, solid black line), for dwarfs in the entire B type domain (Crowther 1998, dashed black line), for Galactic B supergiants (Lefever et al. 2007b, dotted grey line), and for Galactic and SMC B supergiants (Markova \& Puls 2008, solid grey line). Due to wind blanketing effects, the calibrations of the B supergiants lie at lower temperatures than the calibrations of B dwarfs. The supergiants (1.c. I) are indicated by diamonds, the (sub)giants (1.c. II and III) by asterisks and the (sub)dwarfs (1.c. IV and V) by squares. Filled circles indicate the stars for which we do not have any a priori information about luminosity class. It can be seen that spectral types taken from the literature can be quite inaccurate (see text).

reliable than for the bulk of the sample stars. Thus, its derived gravity of 3.70 dex might be compatible with its previous classification as a giant.

Three of the 14 stars with luminosity class IV or V were found to be giants, i.e., they have - within the errorbars - a surface gravity $\log g_{c}$ that is lower than 3.5, i.e., the stars HD 43461 (B6V), HD 50251 (B8V), and HD 182198 (B9V), of which HD 43461 is a rapid rotator and has, therefore, less reliable parameters because of the absence of Si lines. For the other two stars, it is possible that they are rapid rotators, observed almost pole-on. Because of centrifugal effects, the stellar surface would become distorted, and effective temperature (due to gravity darkening) and surface gravity would increase towards the pole. If observed pole-on, both quantities might be underestimated with respect to their average values. On the other hand, the main sequence also becomes extended compared to standard models when rotation is taken into account (e.g., Maeder \& Meynet 2000). Similar results to ours were obtained by Hempel \& Holweger (2003), who found that 6 out of their 27 sample stars with spectral type between B6V and B9V have a $\log g$ below 3.5 .

Depending on the input physics, stellar evolution theory shows that roughly $10-20 \%$ of the B8-B9 stars are in the giant phase, but only 2 to $5 \%$ of B0-B3 stars (e.g., Prialnik 2000; Scuflaire et al. 2008). From the 40 stars without luminosity class treated by us, 11 are found to be outside the main sequence. From the 14 dwarfs, 3 turn out to be giants as well, and from the 10 giants, one was instead found to be a dwarf. Thus our sample contains 23 giants, 19 of which have spectral type B8-B9. Since our sample contains in total 51 stars of spectral type B8B9, this means that the percentage of late type giants in our sample $(37 \%)$ is about twice the expected amount. Only five stars in our sample have spectral types earlier than B3, one of which

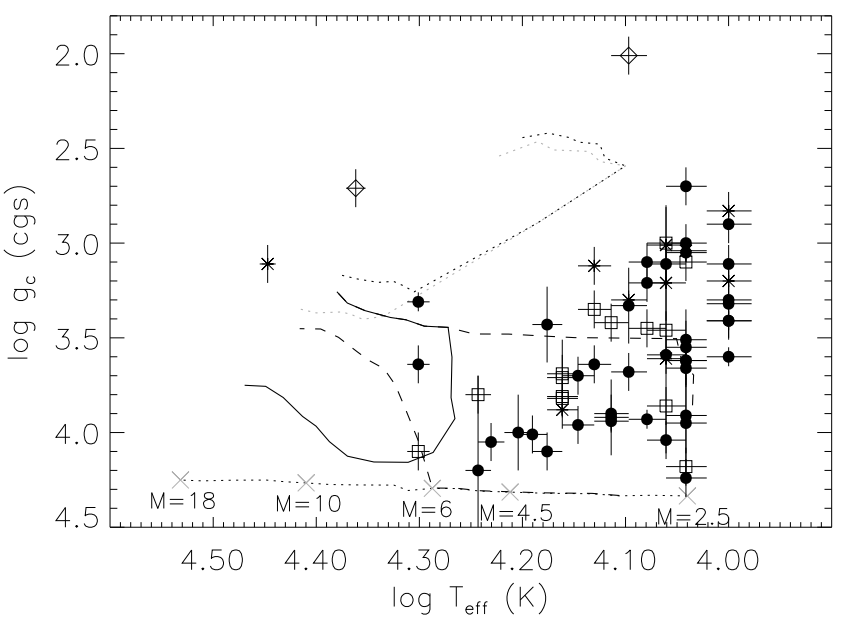

Fig. 10. $\left(T_{\text {eff }}, \log g_{c}\right)$ diagram of the analyzed GAUDI B stars, with the corresponding $2 \sigma$-error bars. Symbols are the same as in Fig. 9. The dotted line represents the ZAMS, and five initial ZAMS masses in $M_{\odot}$ - have been indicated. The most recent theoretical instability domains for the $\beta$ Cephei (thick solid line) and the SPB stars (dashed lines) for main-sequence models, with a core overshooting value of 0.2 times the local pressure scale height, are shown (Miglio et al. 2007a,b), together with the instability domains for post-TAMS models with $\ell=1$ (grey dotted) and $\ell=2$ (black dotted) g-modes computed by Saio et al. (2006). The TAMS is shown as the low-gravity edges of the SPB and $\beta$ Cephei instability domains around $\log g_{c}=3.5$.

is a giant, which provide too low number statistics to verify the expected percentage. We found that 20 of the 40 stars without luminosity class have $v \sin i$ above $90 \mathrm{~km} \mathrm{~s}^{-1}$, and for 8 of them even $v \sin i>150 \mathrm{~km} \mathrm{~s}^{-1}$. This rapid rotation may again explain why we observe twice the number of expected giants when comparing with standard models. Rotation was also found to be a necessary ingredient of evolutionary models when explaining the number of observed giants in the well-studied open clusters $\mathrm{h}$ and $\chi$ Per (Vrancken et al. 2000, in particular their Fig. 3).

\subsection{The macroturbulent and rotational velocities}

When comparing the rotational broadening with macroturbulence, we observe a systematic trend of increasing $v_{\text {macro }}$ with increasing $v \sin i$ for those stars for which some pulsational behavior may be expected, i.e., for the stars where $v_{\text {macro }}$ cannot be neglected (filled circles in Fig. 11). This is consistent with Markova \& Puls (2008, and references therein), who found that, in almost all cases, the size of the macroturbulent velocity was similar to the size of the rotational velocity. On the other hand, they also found $v \sin i$ and $v_{\text {macro }}$ to decrease towards later subtypes, being about a factor of two lower at B9 than at B0.5, and that, in none of their sample stars, rotation alone was able to reproduce the observed line profiles. We cannot confirm either of both statements, because, in contrast, we have found many cases of zero macroturbulence, and many late B type stars with very high macroturbulent velocities. The trend connecting $v \sin i$ and $v_{\text {macro }}$ suggests that it is challenging to differentiate between the effects of both broadening mechanisms.

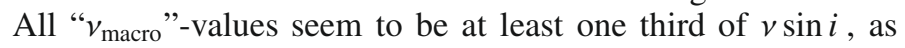
can be inferred from the position of the filled circles above the dashed line in Fig. 11. From a certain projected rotational velocity (i.e., around $v \sin i=120 \mathrm{~km} \mathrm{~s}^{-1}$, dotted vertical line) to larger values, it is impossible to distinguish between both effects, since the pulsational behavior (represented by $v_{\text {macro }}$ ) 


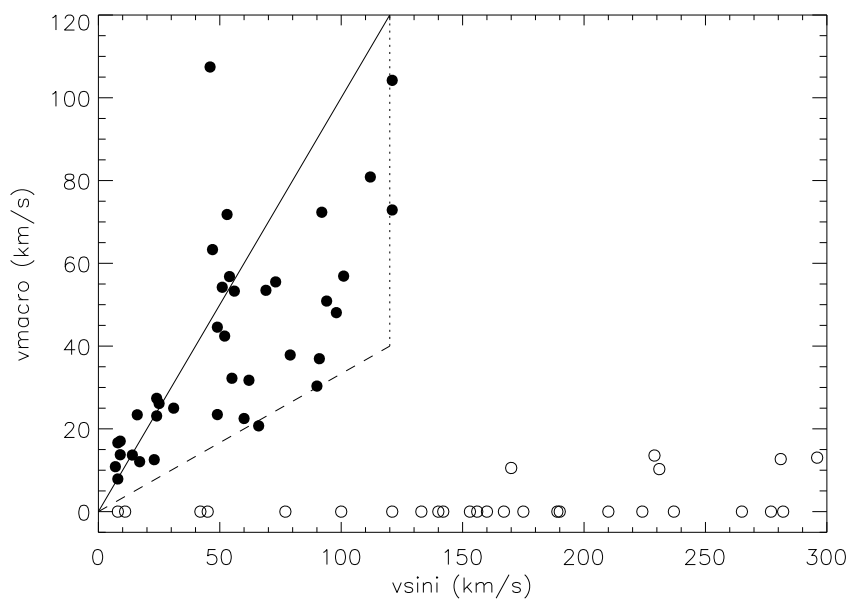

Fig. 11. Comparison between the macroturbulence and the projected rotational velocity $v \sin i$. The solid line is the $1-1$ relation, while the dashed line represents the relation $v_{\text {macro }} / v \sin i=1 / 3$. Stars that we consider as non-pulsating (i.e., which have a negligible $v_{\text {macro }}$ ) are indicated by open circles. The filled circles represent suspected pulsators (i.e., they have a significant $v_{\text {macro }}$ ).

completely disappears in the projected rotational broadening. Following the earlier argumentation that stars that have a nonnegligible macroturbulent velocity may have the largest pulsational amplitudes, we investigated the behavior of $v_{\text {macro }}$ in the $\left(T_{\text {eff }}, \log g\right)$-plane (see Fig. 12). On a global scale, the $v_{\text {macro- }}$ values exhibit a random spread. The position of some individual cases is intriguing, e.g., the supergiant HD $52382\left(\log T_{\text {eff }}\right.$ $\approx 4.36, \log g \approx 2.71$ ). The star's position is compatible with the occurrence of gravity modes excited by the opacity mechanism in evolved stars and may belong to a particular class of pulsating B-type supergiants (Lefever et al. 2007b). As pointed out by Aerts et al. (2009), these gravity-mode oscillations may result in pulsational line broadening, hence the high $v_{\text {macro-value we find. }}$ In addition, HD 48807 (B7 Iab) and HD 48434 (B0 III) show macroturbulence and may belong to the same class. Some of the dwarfs in the sample show macroturbulence, e.g., HD 48215 (B5 V), HD 177880 (B5 V), and HD 50251 (B8 V) might be gmode pulsators in the SPB instability domain. Many stars with $\log g$ below 3.5 have a reasonable to large $v_{\text {macro }}$, which may imply that they are pulsators. It is noteworthy that all instability computations for mid to late B stars so far have been computed for non-rotating models and were stopped artificially at the TAMS from the argument that no star is expected to be found in the Hertzsprung gap. However, B8 to B9 stars transverse this gap at a far lower rate than stars of spectral type earlier than B7 and our observational spectroscopic results point out that it would be worthwhile to extend the instability strip computations beyond the TAMS.

Finally, the magnitude of the microturbulence seems to be randomly distributed in the $\left(T_{\text {eff }}, \log g\right)$-diagram, and related neither to the effective temperature nor the surface gravity (see Fig. 13).

\section{Future use of our results}

The GAUDI database was compiled during the preparation of the CoRoT space mission. We applied our developed automated tool to determine the fundamental parameters to numerous B stars in this database. An independent study used the same database to determine the abundances of the 89 B6-B9 stars in GAUDI, using a different approach based on LTE atmosphere

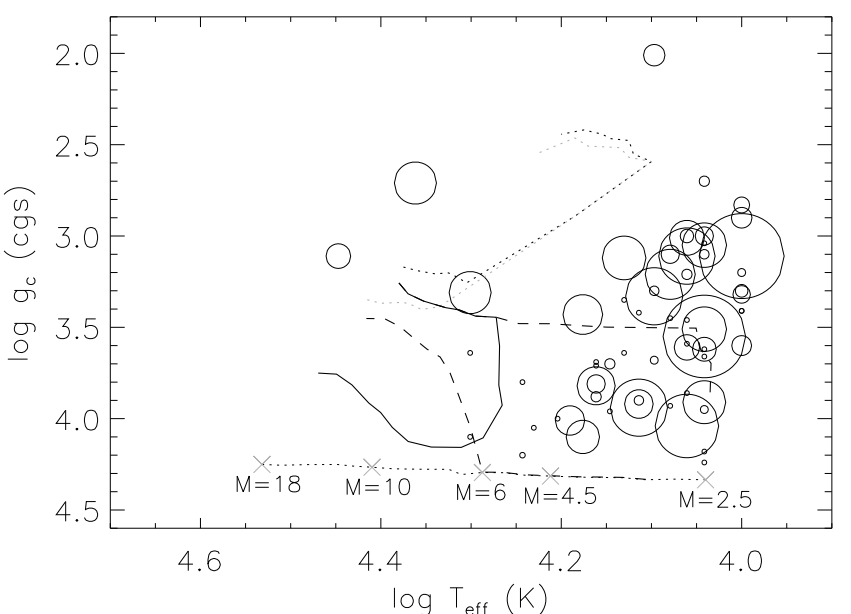

Fig. 12. Same as Fig. 10, but representing the values of $v_{\text {macro }}$ for the GAUDI B star sample. The size of the symbols is proportional to the $v_{\text {macro-value. }}$

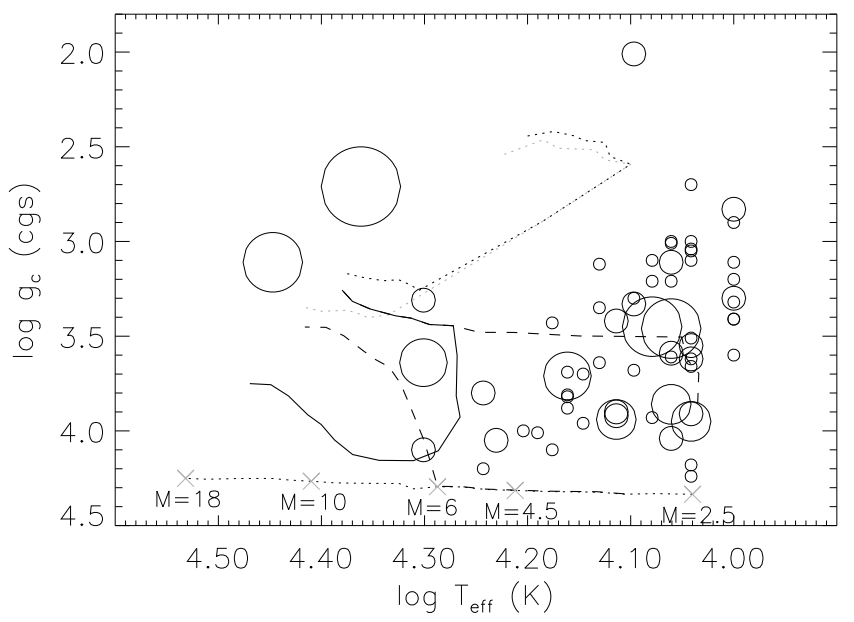

Fig. 13. Same as Fig. 12, but the size of the symbols is now proportional to the microturbulent velocity $\xi$ of the GAUDI B star sample.

models without wind and LTE occupation numbers. We refer to Niemczura et al. (2009) for the underlying assumptions, but mention here that a mild depletion of both $\mathrm{Fe}$ and $\mathrm{Si}$, with average values of the iron and silicon abundances of $7.13 \pm 0.29 \mathrm{dex}$ and $7.22 \pm 0.31$ dex, respectively, was found for their sample. This result was obtained by spectrum synthesis with the LTE approximation, by fixing the effective temperatures from photometric calibrations, the microturbulence to be $2 \mathrm{~km} \mathrm{~s}^{-1}$, and ignoring any macroturbulence in the line profile fits. The mild $\mathrm{Si}$ depletion agrees with our results for the $\mathrm{Si}$ abundance of the late B stars analyzed by ourselves.

The first set of CoRoT data have been released to the public. Two of our sample stars have already been observed by the satellite, i.e., HD 181440 (B9 III) and HD 182198 (B9 V). The spectra of these stars were available in the GAUDI database and their parameters are listed in Table 3. Their seismic analysis is under way (Miglio et al., in preparation).

We emphasize that one of the primary targets of CoRoT is HD 180642 (B1.5 II-III), which is a mildly N-enriched, apparently slowly rotating $\beta$ Cephei star, discovered by Aerts (2000). Morel \& Aerts (2007) determined its fundamental parameters from high-resolution spectroscopy and derived $T_{\mathrm{eff}}=$ $24500 \pm 1000 \mathrm{~K}, \log g=3.45 \pm 0.15$, and $\xi=12 \pm 3 \mathrm{~km} \mathrm{~s}^{-1}$. 
By applying AnalyseBstar, we found that the model with $T_{\text {eff }}=$ $24000 \pm 1000 \mathrm{~K}, \operatorname{logg}=3.40 \pm 0.10$, and $\xi=13 \pm 2 \mathrm{~km} \mathrm{~s}^{-1}$ is the closest fitting, so our results agree very well with theirs. We thus classify this star as a pulsator very close to the TAMS. It will be very interesting to see whether this spectroscopic result is confirmed by the seismic modeling of oscillation frequencies derived from the CoRoT space photometry, because this would imply that one of the few $\beta$ Cephei stars is very close to the end of the core-hydrogen burning stage.

At the time of launch, the CoRoT space mission was nominally approved to operate only until 2009 , but, given its excellent performance, the operation time will probably be extended. It is therefore to be expected that several more B-type stars in GAUDI will be observed by the mission in the near future.

Acknowledgements. The research leading to these results has received funding from the European Research Council under the European Community's Seventh Framework Programme (FP7/2007-2013)/ERC grant agreement no 227224 (PROSPERITY), as well as from the Research Council of K.U. Leuven grant agreement GOA/2008/04. The authors wish to thank Alex de Koter cordially for insightful discussions and helpful comments throughout the project. The authors would like to thank the anonymous referee for valuable comments and suggestions on how to improve this paper.

\section{Appendix A: Formal convergence tests for synthetic FASTWIND spectra - some details}

Before applying AnalyseBstar to real, observed spectra, we first tested whether the method is able to recover the input parameters of synthetic spectra. For this purpose, we created several synthetic datasets in various regions of parameter space. We do not discuss all of them here, but we chose three specific examples (lying exactly on a grid point), each representative of a different type of star:

- Dataset A: a B0.5 I star with a rather dense stellar wind and a strongly enhanced helium abundance.

- Dataset B: a B3 III star with a weak stellar wind and a depleted $\mathrm{Si}$ abundance.

\section{- Dataset C: a B8 V with a very thin stellar wind.}

Each dataset was convolved with both a rotational and a macroturbulent broadening profile. The projected rotational velocity adopted is $50 \mathrm{~km} \mathrm{~s}^{-1}$ for each dataset, characteristic of a "typical" slow rotator. In the case of low and intermediate resolution spectra, there is also the possibility to carry out an additional Gaussian, instrumental convolution. This is, however, not necessary for spectra of such high resolution as that of FEROS and ELODIE. Artificial (normally distributed) noise was added to mimic a true spectrum with a mean local SNR of 150, which is more or less the minimal local SNR obtained for the GAUDI sample.

Finally, we prepared the spectra as if they were real data, i.e., defining the EW of all available lines, measuring the SNR to account for the errors in the EW and fixing the projected rotational velocity. Since we analyze synthetic data, obviously no normalization was required.

Table A.1 lists the input parameters of the three synthetic datasets as well as the output parameters, obtained applying AnalyseBstar. For each dataset, the second column gives the derived "interpolated" values, while the third column lists the parameters of the closest (best-fit) grid model. In the ideal case, both should be exactly the same as the input parameters. This depends, however, on how well the equivalent widths were measured and minor deviations occur as expected.

Besides these three cases, in which we started from a synthetic model given by one of the grid points, we additionally created three synthetic datasets in which the parameters lie inbetween different grid points. In analogy to datasets $A$ to $C$, their profiles were convolved with both a rotational and macroturbulent broadening profile and artificial noise was added. The input parameters and the results of the analysis were added to Table A.1 as datasets D, E, and F.

A third set of models $(\mathrm{G}, \mathrm{H}, \mathrm{I})$ consists of models computed for parameters not included in the grid, but within the grid limits, and this time without noise, with the aim of testing the predictive power of our method, independent of the source of errors introduced by the noise. The rotational velocity applied to these test models is $30 \mathrm{~km} \mathrm{~s}^{-1}$, and the macroturbulent velocity is $15 \mathrm{~km} \mathrm{~s}^{-1}$. We find an overall very good agreement between the closest fitting grid model and the interpolated values, and the input parameters.

Although the models given in Table A.1 were selected to be the best fitting models, the program additionally came up with some other models, which agree with the input model within the errors, introduced by the artificial noise and the errors in the determination of the equivalent widths (datasets A to F) and/or the various interpolations (datasets D to I). For instance, for dataset I, AnalyseBstar came up with a second possibility with slightly different parameters, even closer to the input data: $\log g=1.7, \xi=6.5 \mathrm{~km} \mathrm{~s}^{-1}, \nu_{\text {macro }}=14.6 \mathrm{~km} \mathrm{~s}^{-1}$, $N(\mathrm{He}) / N(H)=0.12$, but with $\log n(\mathrm{Si}) / n(\mathrm{H})=-4.49$ (the other parameters are the same as those for the model in the table). Since the Si abundance is overestimated compared to its input value, the $\mathrm{Si}$ lines are overall a bit too strong and the final fit is slightly poorer than for the model in the table.

In all nine cases, the input parameters are well recovered. Typically 10 to 150 models are selected and considered during the analysis cycle. The number depends not only on the choice of the initial parameters, but also on the accuracy of the measured quantities and the procedure applied (hence, the temperature domain, see below). The longer computation times for the cooler models are caused by the different method applied in this range. We considered numerous other test cases, which are not included in this text, but for which the input parameters were equally well recovered. Slight deviations from the input parameters are as expected. In the cool temperature domain (datasets $\mathrm{C}, \mathrm{F}$, and I), where our alternative optimization "method 2" is required (see Sect. B.3), we are also still able to deduce reliable parameter estimates, at least for the synthetic models. The results for the Si abundance in datasets F clearly show the problem for estimating the abundances when only one ion is present: the estimation of the Si abundance was, for this particular test case, not equal to the input value. When we have only Si II lines, the results cannot be as secure as when we have two ionisation stages of $\mathrm{Si}$. This problem was accounted for by adopting somewhat larger errors in the derived parameters, for objects with $T_{\text {eff }}<15000 \mathrm{~K}$ (see Table 3).

When the observed object is close to one of the borders of the grid, the method assumes "the border" to be the closest match. This will, consequently, lead to larger errors in some of the other parameters.

The derived $v \sin i$-value, which is difficult to distinguish from the macroturbulent velocity, agrees very well with the inserted values, irrespective of $v_{\text {macro }}$. This shows that the Fourier transform method indeed allows us to separate both effects. Altogether, this provides us with confidence that our method is 
Table A.1. Input parameters for nine synthetic models (IN) are compared to the actual (interpolated and best-fit model) output parameters (OUT) obtained through the application of AnalyseBstar to these synthetic data (see text for further explanation and notes to this table for the meaning of the symbols).

\begin{tabular}{|c|c|c|c|c|c|c|c|c|c|}
\hline$a$ & IN & $\begin{array}{r}\text { OUT } \\
\text { inter- } \\
\text { olated }^{b}\end{array}$ & $\begin{array}{l}\text { OUT } \\
\text { grid }\end{array}$ & IN & $\begin{array}{r}\text { OUT } \\
\text { inter- } \\
\text { polated }\end{array}$ & $\begin{array}{r}\text { OUT } \\
\text { grid }\end{array}$ & IN & $\begin{array}{r}\text { OUT } \\
\text { inter- } \\
\text { polated }\end{array}$ & $\begin{array}{l}\text { OUT } \\
\text { grid }\end{array}$ \\
\hline Fit parameter & \multicolumn{3}{|c|}{ Dataset A } & \multicolumn{3}{|c|}{ Dataset B } & \multicolumn{3}{|c|}{ Dataset C } \\
\hline$T_{\text {eff }}(\mathrm{K})$ & 23,000 & 22,600 & 23,000 & 18,000 & 18,100 & 18,000 & 13,000 & 13,000 & 13,000 \\
\hline $\log g(\mathrm{cgs})$ & 2.7 & 2.7 & 2.7 & 3.3 & 3.3 & 3.3 & 4.2 & 4.2 & 4.2 \\
\hline $\log Q(\text { char })^{c}$ & $\mathrm{C}$ & C & $\mathrm{C}$ & A & $b$ & $\mathrm{~b}$ & $\mathrm{O}$ & $a$ & a \\
\hline$\beta$ & 2.0 & 2.0 & 2.0 & 1.5 & 1.2 & 1.2 & 0.9 & 0.9 & 0.9 \\
\hline$n(\mathrm{He}) / n(\mathrm{H})$ & 0.20 & 0.18 & 0.20 & 0.10 & 0.08 & 0.10 & 0.10 & 0.08 & 0.10 \\
\hline $\log n(\mathrm{Si}) / n(\mathrm{H})$ & -4.49 & -4.49 & -4.49 & -4.79 & -4.81 & -4.79 & -4.79 & -4.79 & -4.79 \\
\hline$\xi\left(\mathrm{km} \mathrm{s}^{-1}\right)$ & 10 & 10.2 & 10 & 15 & 15 & 15 & 6.0 & 7.3 & 6.0 \\
\hline$v \sin i\left(\mathrm{~km} \mathrm{~s}^{-1}\right)$ & 50 & $48 \pm 2$ & $48 \pm 2$ & 50 & $48 \pm 4$ & $48 \pm 4$ & 50 & $51 \pm 1$ & $51 \pm 1$ \\
\hline$v_{\text {macro }}\left(\mathrm{km} \mathrm{s}^{-1}\right)$ & 20 & $29 \pm 4$ & $29 \pm 4$ & 30 & $33 \pm 2$ & $33 \pm 2$ & 10 & $30 \pm 1$ & $30 \pm 1$ \\
\hline time (s) & 142 & & & 494 & & & 2344 & & \\
\hline \multirow[t]{2}{*}{ checked models } & 10 & & & 21 & & & 128 & & \\
\hline & \multicolumn{3}{|c|}{ Dataset D } & \multicolumn{3}{|c|}{ Dataset E } & \multicolumn{3}{|c|}{ Dataset F } \\
\hline$T_{\text {eff }}(\mathrm{K})$ & 21,120 & 20,985 & 21,000 & 15,100 & 15,030 & 15,000 & 11,880 & 11,500 & 11,500 \\
\hline $\log g(\mathrm{cgs})$ & 3.98 & 4.0 & 4.0 & 1.83 & 1.80 & 1.8 & 2.43 & 2.3 & 2.3 \\
\hline $\log Q$ (char) & $\mathrm{b}$ & $b$ & $\mathrm{~b}$ & A & $A$ & A & $\mathrm{a}$ & $O$ & $\mathrm{O}$ \\
\hline$\beta$ & 1.42 & 2.0 & 1.2 & 2.8 & 3.0 & 3.0 & 1.02 & 0.9 & 0.9 \\
\hline$n(\mathrm{He}) / n(\mathrm{H})$ & 0.14 & 0.11 & 0.10 & 0.08 & 0.09 & 0.10 & 0.18 & 0.20 & 0.20 \\
\hline $\log n(\mathrm{Si}) / n(\mathrm{H})$ & -4.85 & -4.80 & -4.79 & -4.23 & -4.16 & -4.19 & -4.49 & -4.19 & -4.19 \\
\hline$\xi\left(\mathrm{km} \mathrm{s}^{-1}\right)$ & 12 & 15.7 & 15 & 11 & 10.6 & 10 & 9 & 6.3 & 6 \\
\hline$v \sin i\left(\mathrm{~km} \mathrm{~s}^{-1}\right)$ & 50 & $52 \pm 6$ & $52 \pm 6$ & 50 & $50 \pm 7$ & $50 \pm 7$ & 50 & $54 \pm 1$ & $54 \pm 1$ \\
\hline$v_{\text {macro }}\left(\mathrm{km} \mathrm{s}^{-1}\right)$ & 40 & $33 \pm 5$ & $33 \pm 5$ & 10 & $39 \pm 7$ & $39 \pm 7$ & 70 & $67 \pm 7$ & $67 \pm 7$ \\
\hline time (s) & 174 & & & 392 & & & 1254 & & \\
\hline \multirow[t]{2}{*}{ checked models } & 10 & & & 26 & & & 95 & & \\
\hline & \multicolumn{3}{|c|}{ Dataset $\mathrm{G}$} & \multicolumn{3}{|c|}{ Dataset $\mathrm{H}$} & \multicolumn{3}{|c|}{ Dataset I } \\
\hline$T_{\mathrm{eff}}(\mathrm{K})$ & 24,800 & 24,650 & 25,000 & 18,600 & 18,392 & 18,500 & 10,910 & 11,000 & 11,000 \\
\hline $\log g(\mathrm{cgs})$ & 3.33 & 3.3 & 3.3 & 2.78 & 2.8 & 2.8 & 1.72 & 1.8 & 1.8 \\
\hline $\log Q$ (char) & B & $B$ & B & $\mathrm{O}$ & $A$ & A & $\mathrm{C}$ & $C$ & $\mathrm{C}$ \\
\hline$\beta$ & 1.1 & 1.2 & 1.2 & 1.0 & 0.9 & 0.9 & 2.10 & 2.0 & 2.0 \\
\hline$n(\mathrm{He}) / n(\mathrm{H})$ & 0.11 & 0.12 & 0.10 & 0.14 & 0.13 & 0.15 & 0.11 & 0.09 & 0.10 \\
\hline $\log n(\mathrm{Si}) / n(\mathrm{H})$ & -4.52 & -4.48 & -4.49 & -4.25 & -4.22 & -4.19 & -4.67 & -4.79 & -4.79 \\
\hline$\xi\left(\mathrm{km} \mathrm{s}^{-1}\right)$ & 13 & 13.5 & 12 & 4 & 3.3 & 3 & 7 & 8.6 & 10 \\
\hline$v \sin i\left(\mathrm{~km} \mathrm{~s}^{-1}\right)$ & 30 & $30 \pm 1$ & $30 \pm 1$ & 30 & $30 \pm 1$ & $30 \pm 1$ & 30 & $30 \pm 1$ & $30 \pm 1$ \\
\hline$v_{\text {macro }}\left(\mathrm{km} \mathrm{s}^{-1}\right)$ & 15 & $12 \pm 4$ & $12 \pm 4$ & 15 & $20 \pm 7$ & $20 \pm 7$ & 15 & 0 & 0 \\
\hline time $(\mathrm{s})$ & 2119 & & & 2284 & & & 356 & & \\
\hline checked models & 66 & & & 67 & & & 15 & & \\
\hline
\end{tabular}

Notes. ${ }^{(a)} T_{\text {eff }}$ - Effective temperature, $\log g$ - surface gravity, $\log Q$ - wind strength parameter (see text), $\beta$ - wind velocity exponent, $n(\mathrm{He}) / n(\mathrm{H})-$ He abundance, $\log n(\mathrm{Si}) / n(\mathrm{H})-\mathrm{Si}$ abundance, $\xi$ - microturbulent velocity, $v \sin i$ - projected rotational velocity, $v_{\text {macro }}-$ macroturbulent velocity, time elapsed in seconds during the run of AnalyseBstar, and number of different models that have been checked throughout the procedure. ${ }^{(b)}$ For $\log g, \log Q$ and $\beta$ no real interpolation was made. ${ }^{(c)}$ In our grid, we allow for 7 different values for $\log Q$, represented by a character: $\log Q=$ -14.30 (O), -14.00 (a), -13.80 (A), -13.60 (b), -13.40 (B), -13.15 (C), -12.70 (D).

working reliably and that the procedure will also be able to recover the true physical parameters from real spectra.

The macroturbulent velocity is the only fit parameter for which significant deviations arise, if $v_{\text {macro }}$ is low $\left(<30 \mathrm{~km} \mathrm{~s}^{-1}\right)$.
However, larger macroturbulences are recovered well. For datasets $\mathrm{C}, \mathrm{E}$, and I, where $v_{\text {macro }}$ is only $10-15 \mathrm{~km} \mathrm{~s}^{-1}$, the deviation is particularly large (of the order of between 15 and $30 \mathrm{~km} \mathrm{~s}^{-1}$ ). To understand this discrepancy, we first considered 

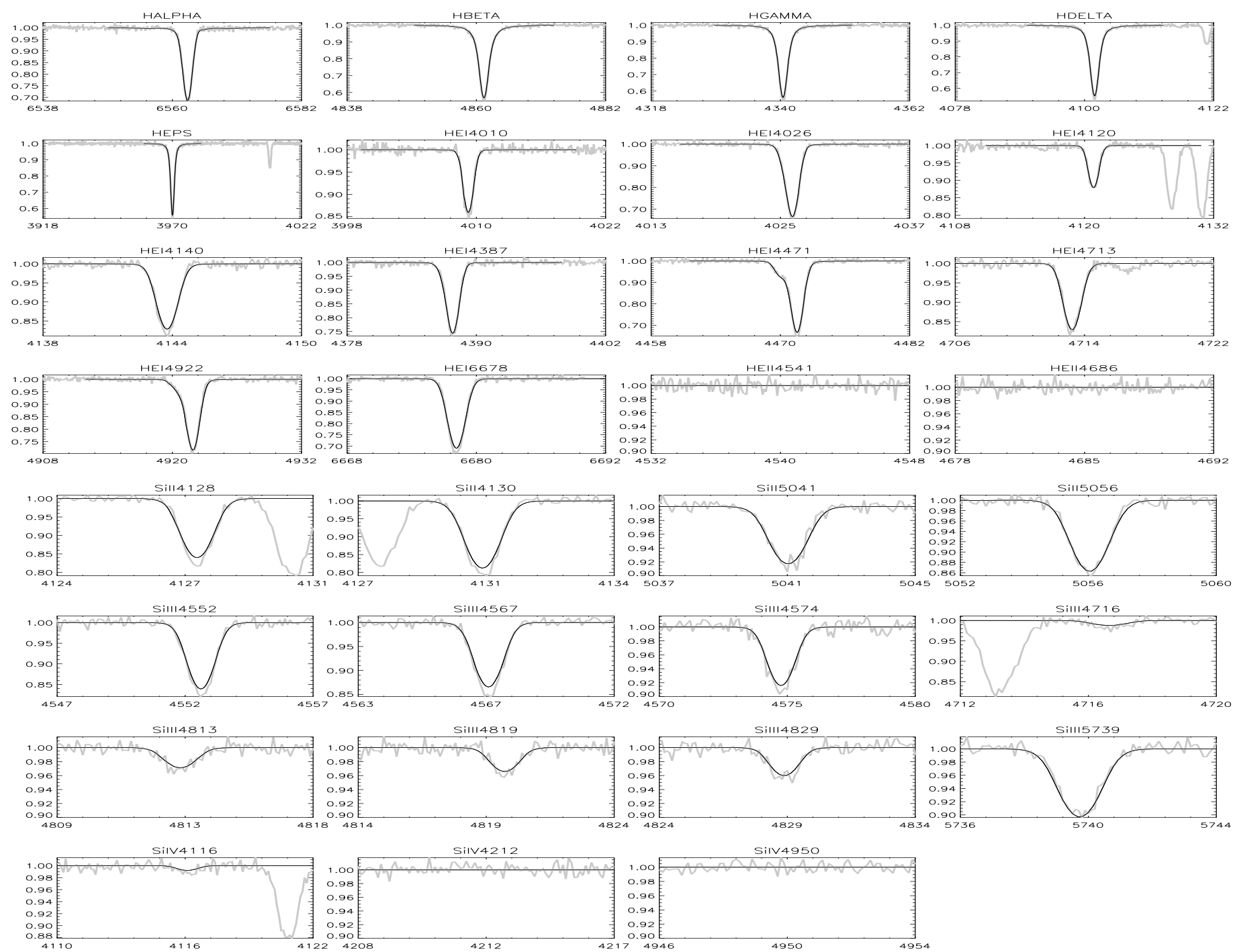

Fig. A.1. Example of the fit quality for dataset E. The slight discrepancy in the cores of the Si lines arises from the difference in Si abundance between the interpolated value $(\log n(\mathrm{Si}) / n(\mathrm{H})=-4.16)$ and the closest grid value $(\log n(\mathrm{Si}) / n(\mathrm{H})=-4.19)$.

the line profiles, which show that the fit is almost perfect for this $v_{\text {macro }}$ (see Fig. A.1). After verifying the fit quality, we also verified that the discrepancies do not arise from our applied procedure, by performing several tests on simulated spectra, in which we left only $v_{\text {macro }}$ as a free parameter. In all cases, the macroturbulent velocity was recovered very well, with deviations within $5 \mathrm{~km} \mathrm{~s}^{-1}$. The (sometimes significant) deviations in $v_{\text {macro }}$ can be understood as a compensation for differences between the interpolated values of the "observed" line profiles and the closest grid model. Indeed, as shown by Aerts et al. (2009), small deviations in the wings of the $\mathrm{Si}$ profiles can result in wrong estimates of $v_{\text {macro }}$ and $v \sin i$, because these two velocity fields are difficult to differnetiate between whenever one of them is small. Thus, the values derived for $v_{\text {macro }}$ should be treated with caution. This does not affect the derivation of the other parameters, however, since a convolution with the $v_{\text {macro-profile preserves the } \mathrm{EW} \text { of }}$ the lines. It is the EW, and not the line profile shape, that is used in the derivation of $T_{\text {eff }}, \xi$, and $\log n(\mathrm{Si}) / n(\mathrm{H})$. The other parameters (gravity, mass-loss rate, and wind velocity law) remain unaffected because they are inferred from the Balmer lines, which are not very sensitive to the $v_{\text {macro }}$ values due to the dominance of the Stark broadening. Thus, we find a very good agreement between their input and output values (Table A.1).

\section{Appendix B: Methodology of AnalyseBstar}

In what follows, we provide a detailed description of AnalyseBstar. In Fig. B.1, a flowchart of the full program is drawn schematically. The reader is advised to follow this context diagram throughout the additional description of AnalyseBstar.

\section{B.1. Preparation of the input}

No automatic method can fully replace the "by-eye" procedure, and this is also true for AnalyseBstar. A few steps still require human intervention, in particular the preparation of the spectra and the input to the main program, as we show in the following.

Observation of peculiarities. The first required step when starting the analysis is to inspect the spectrum for any "abnormalities", such as line behavior resulting from nebulae, disks, binarity, or other peculiarities. Special features can and should be detected by eye inspection.

Merging and normalization of the spectra. When dealing with échelle spectra, such as the ELODIE and FEROS spectra in the 


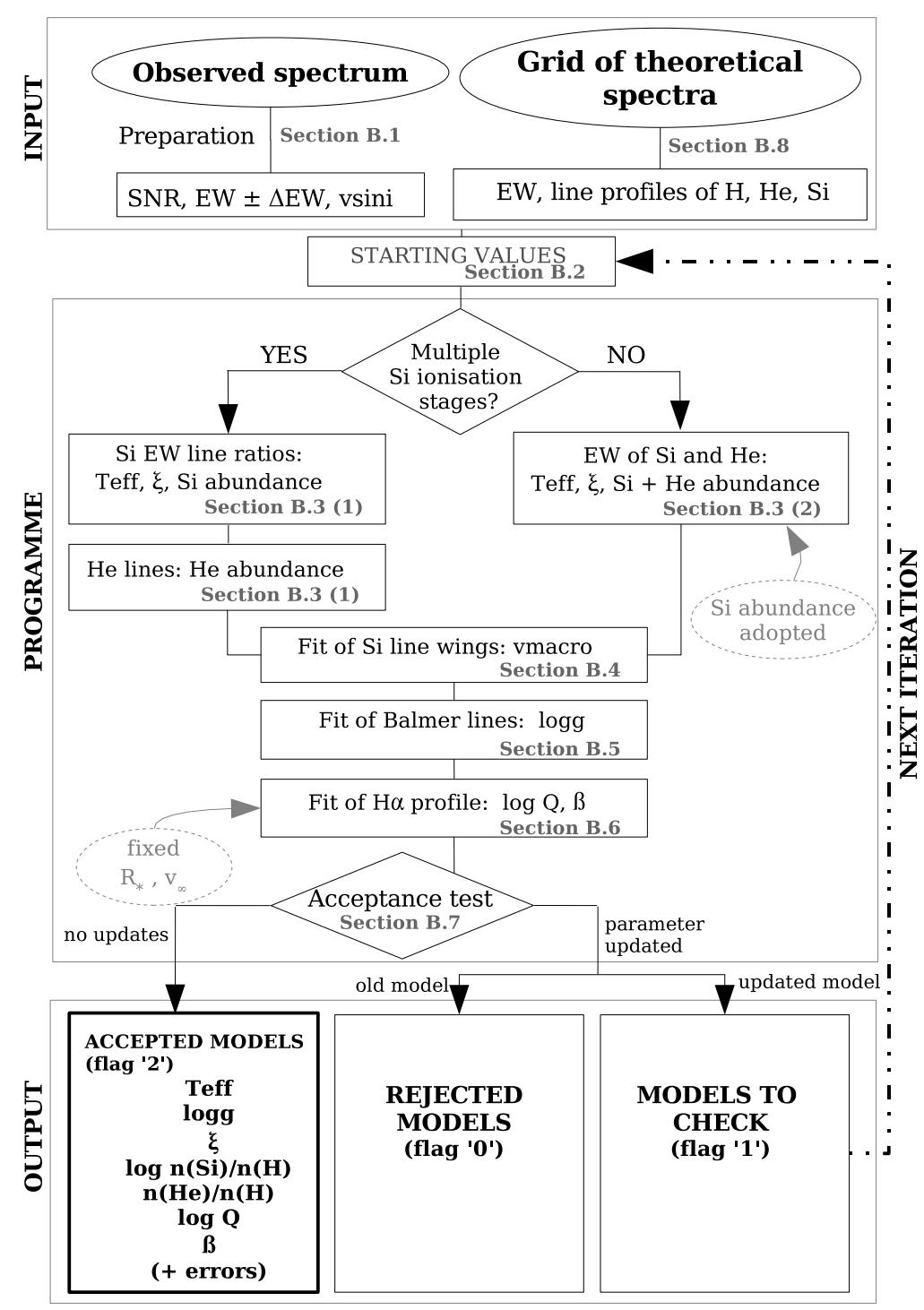

Fig. B.1. Context diagram of AnalyseBstar. For a detailed explanation, see text.

GAUDI database, we first need to merge the spectral orders before the spectrum can be normalized. This is a very delicate and non-trivial process. The edges of the orders are often noisy, due to the decrease in sensitivity of the CCD near its edges, and are therefore removed. Spectral lines falling inconveniently at the edge of one spectral order or in the overlap region of two consecutive orders, thus producing a cut-off to the spectral line, are not reliable for deriving accurate stellar information. They can, in the best case, only be used afterwards as a consistency check. After the spectra have been merged, they are normalized by interactive interpolation of continuum regions. This is performed over a wide wavelength coverage, where sufficient continuum regions are present, to avoid line corruption that can propagate into measurement of the fundamental parameters. The normalization is particularly important to the determination of the gravity, which depends sensitively on the shape of the Balmer line wings.

Radial velocity correction. The spectra need to be corrected for radial velocity shifts. Shifting the spectrum to restframe wavelengths automatically has not yet been included in the current version.
Line selection. As a standard procedure, we infer a number of line profiles in the optical from the underlying model atmospheres. These standard line profiles can be used directly. Computing additional line profiles is, however, straightforward. The selection of lines to be used during the spectral analysis in AnalyseBstar is gathered from a file that contains a default (but easily editable) list of currently used optical lines (see below). We distinguish between optical lines that will be used to predict the physical parameters during the spectral line fitting procedure, and those that will not be used explicitly for reasons of uncertainties, either in the theoretical predictions or in the observed spectrum (due to problems in merging or normalization). The goodness-of-fit of the latter set of lines is checked only to investigate systematic differences between theory and observations, which can be useful when considering additional improvements to the atomic data and/or atmospheric models. These lines are $\mathrm{H} \epsilon$, He I 4010, 4120, 4140, and Si IV 4950. The lines that will be used in the fitting procedure because of their predictive power are the following: the Balmer lines $\mathrm{H} \alpha, \mathrm{H} \beta, \mathrm{H} \gamma$ and $\mathrm{H} \delta$, the neutral and singly ionized He lines He I 4026, 4387, 4471, 4713, 4922, 6678, He II 4541 and He II 4686, and the Si lines in their three different ionization stages Si II 4128-4130, Si II 5041-5056, 
Si III 4552-4567-4574, 4716, 4813-4819-4829, 5739, Si IV 4116 , and Si IV 4212 . He II 4200 is excluded because it can only safely be used for O-type stars and not for B-type stars. At least for dwarfs, it only appears at temperatures above about $25000 \mathrm{~K}$, where it is still overruled by N II, while at hotter temperatures, He II 4200 is for $50 \%$ blended by $\mathrm{N} \mathrm{III}^{7}$.

Signal-to-noise measurement. To estimate the error in the equivalent width as accurately as possible, we need to determine the SNR. Since the SNR can vary a lot with wavelength, we chose to calculate the local SNR of each line separately, by using a continuum region close to the line. This is performed manually by indicating the left and right edges of the continuum interval. The SNR is then given by the mean flux $\overline{F_{c}}$ divided by the standard deviation $\sigma\left(F_{c}\right)$ of the flux in this interval, i.e.,

$\mathrm{SNR}=\overline{F_{c}} / \sigma\left(F_{c}\right)$.

Observed equivalent widths and their errors. (cf. Fig. B.2). The equivalent widths of most $\mathrm{He}$ and $\mathrm{Si}$ lines can be measured by a Gaussian non-linear least squares fit to the observed line profiles, using the Levenberg-Marquardt algorithm (Levenberg 1944; Marquardt 1963). We thoroughly tested whether this was a justifiable approach by comparing, for multiple line profiles of stars with completely different properties, the equivalent widths computed from a Gaussian fit to the observed line profiles with those computed from a proper integration of the line pixel values. This comparison resulted in a minimal difference between both approaches. Some lines obviously cannot be fitted by a Gaussian, e.g., the He lines with a strong forbidden component in their blue wing or Stark-broadened lines such as those from hydrogen. The EW of these lines are determined by proper integration. In all other cases, where the line profile $i s$ represented well by a Gaussian fit, the observed EW is given by the integral of the Gaussian profile.

The main source of uncertainty in the EW determination is introduced by the noise and its influence on the exact position of the continuum within the noise. To account for this, we multiplied the continuum by a factor $(1 \pm 1 / \mathrm{SNR})$, performed a new Gaussian fit to both shifted profiles and measured the difference between the EWs. Note that, by considering a constant factor over the full line profiles (using the signal at continuum level), we implicitly place equal weight on each wavelength point, even though, in reality, 1/SNR may be slightly higher in the cores, since it is proportional to $\sqrt{1 / S}$, where $S$ is the obtained signal at these wavelengths. When the user deems it necessary, (s)he can apply a correction to the continuum level using a local rerectification, e.g., in the case that the normalization performed over a wide wavelength range is locally a little offset. If the offset is different on the blue and red side of the line, then the factor should be wavelength dependent. Therefore, we allow for a linear rerectification by a factor $a \lambda+b$. Another source of uncertainty in the EW is introduced by using a Gaussian fit to estimate the EW. Each of the above factors were included in the total error budget of the EW.

Determination of $v \sin i$. (See Fig. B.3). The determination of the projected rotational velocity is the last step in the preparation of the input for AnalyseBstar. We use the semi-automatic tool developed by Simón-Díaz \& Herrero (2007) to derive

\footnotetext{
7 See http://www.lsw.uni-heidelberg.de/cgi-bin/ websynspec.cgi
}

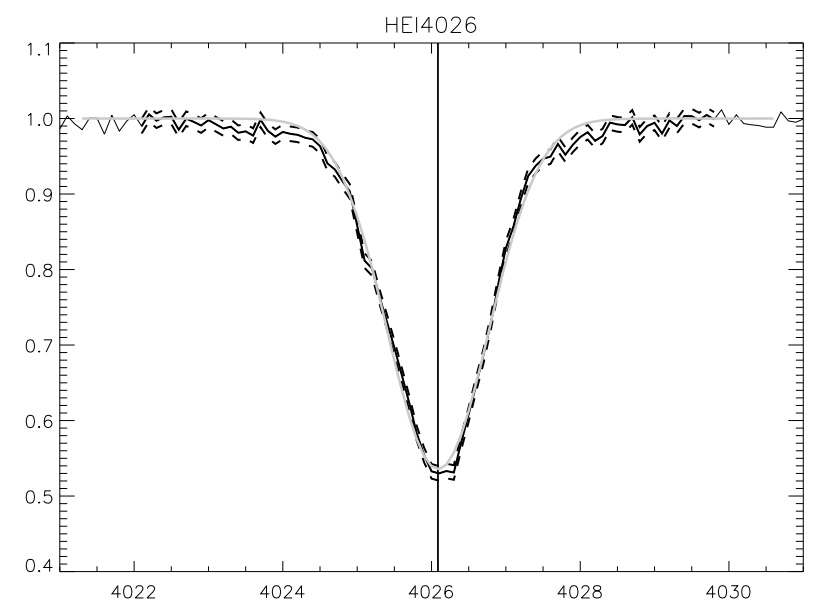

Fig. B.2. Illustration of the EW determination for He I 4026. After the manual identification of the wavelength interval of the spectral line (thick black profile), a Gaussian fit to the observed line profile is made to determine the EW of the line (thick grey profile). Also indicated are: the center of the line (vertical line) and the "shift" in flux, upwards and downwards (dashed line profiles), used to account for the noise level in the determination of the EW. A Gaussian fit was made also to these line profiles, but these are omitted here for the sake of clarity.

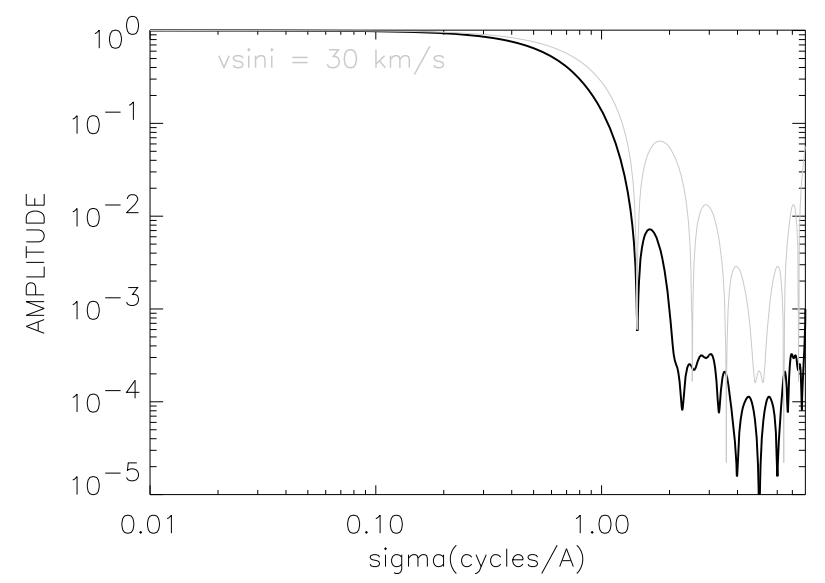

Fig. B.3. Illustration of the determination of $v \sin i$ from the first minimum in the Fourier transform of the selected line profile, as implemented and described by Simón-Díaz \& Herrero (2007). The user can make several attempts and finally decide which $v \sin i$ gives the best match. The difference between the slopes for the first decay gives an indication of the macroturbulence. The Fourier transform of the observed line profile is indicated in black. The user indicates the first minimum in the black profile, which gives the projected rotational velocity. The Fourier transform of the rotational profile at this vsini is indicated in grey.

the projected rotational velocities (see Fig. B.3). We refer to their paper for a thorough discussion. We use the following, least blended, metallic lines for the determination of $v \sin i$ : Si II 5041, Si III 4567, 4574, 4813, 4819, 4829, 5739, C II 4267, $6578,6582,5133,5145,5151$, and O II 4452. We carefully checked visually that blended lines were not taken into account. The projected rotational velocity $v \sin i$ of the star is calculated as the mean of the values derived from each individual line, and its uncertainty as the standard deviation. 


\section{B.2. Fit parameters and their starting values}

Once all preparation is finished, the automatic procedure to obtain accurate values of all physical fit parameters can be started. This procedure follows an iterative scheme as outlined in Fig. B.1. In each iteration, the fundamental parameters are improved in the following order. First of all, the effects of the effective temperature, the Si abundance, and the microturbulence on the $\mathrm{Si}$ lines are separated. The He abundance is then fixed using all available He lines. Next, the macroturbulent velocity is determined from well-chosen, user-supplied Si lines. The surface gravity is then determined from the wings of $\mathrm{H} \gamma, \mathrm{H} \delta$, and, in the case of weak winds, also $\mathrm{H} \beta$, after which the wind strength $\log Q$ ( $Q=\dot{M} /\left(v_{\infty} R_{*}\right)^{1.5}$, where $\dot{M}$ is the mass loss, $v_{\infty}$ is the terminal wind velocity, and $R_{*}$ is the stellar radius) and the wind velocity exponent $\beta$ are determined in parallel using $\mathrm{H} \alpha$. Obviously, to begin the iterative procedure, we need an initial guess of each of these parameters. This initial value can either be user supplied or standard. If no value is set by the user, then the following initial values are considered. The initial effective temperature will be determined from the spectral type of the star. Immediately after the rotational and macroturbulent velocities have been determined, a start value for the surface gravity is derived by running the subprocedure for the determination of the gravity (see Sect. B.5). For the He and the Si abundance, we have taken the lowest value as a start value. We initialize the wind parameters at their lowest possible values, which corresponds to negligible wind, at $\log Q=-14.30$ and $\beta=0.9$. These are reasonable assumptions for dwarfs, which represent the largest part of our sample. These starting values can easily be adapted when samples of stars with different stellar properties are targeted. The macroturbulent velocity is initially zero, whereas for the microturbulent velocity we assume a medium value of all considered possibilities, i.e., $10 \mathrm{~km} \mathrm{~s}^{-1}$. The convergence speed depends on how far away the initial values are from the final solution.

\section{B.3. Determination of the effective temperature, microturbulence, and abundances}

The use of Si lines has multiple purposes. By using a welldefined scheme (somewhat similar to the conventional "curve of growth" method (Gray 1976) and described, e.g., by Urbaneja 2004), we are able to separate the effects of the effective temperature, the Si abundance, and the microturbulence on the line profiles, and derive an accurate value for them. Our method automatically determines from the observed spectrum which lines will be used for this purpose. The lines should be clearly visible (i.e., clearly distinguishable from the noise in the continuum) and the relative error in their equivalent width is typically below 10 to $15 \%$, depending on the combination of the temperature and the specific line considered. Whenever the relative error in its equivalent width exceeds $75 \%$, the spectral line is excluded in the following procedures. Such large errors are a rare exception and only occur for the weakest lines, which are indistinguishable from the noise level.

We can basically discern two separate cases, namely when multiple and consecutive ionization stages of $\mathrm{Si}$ are available, or when there is only one ionization stage of Si. Each needs a different approach, as we now explain.

Method 1: When multiple ionization stages of Si are available. We can, to first order, put aside the effect of the Si abundance by considering the EW ratios of two different Si ionization stages,
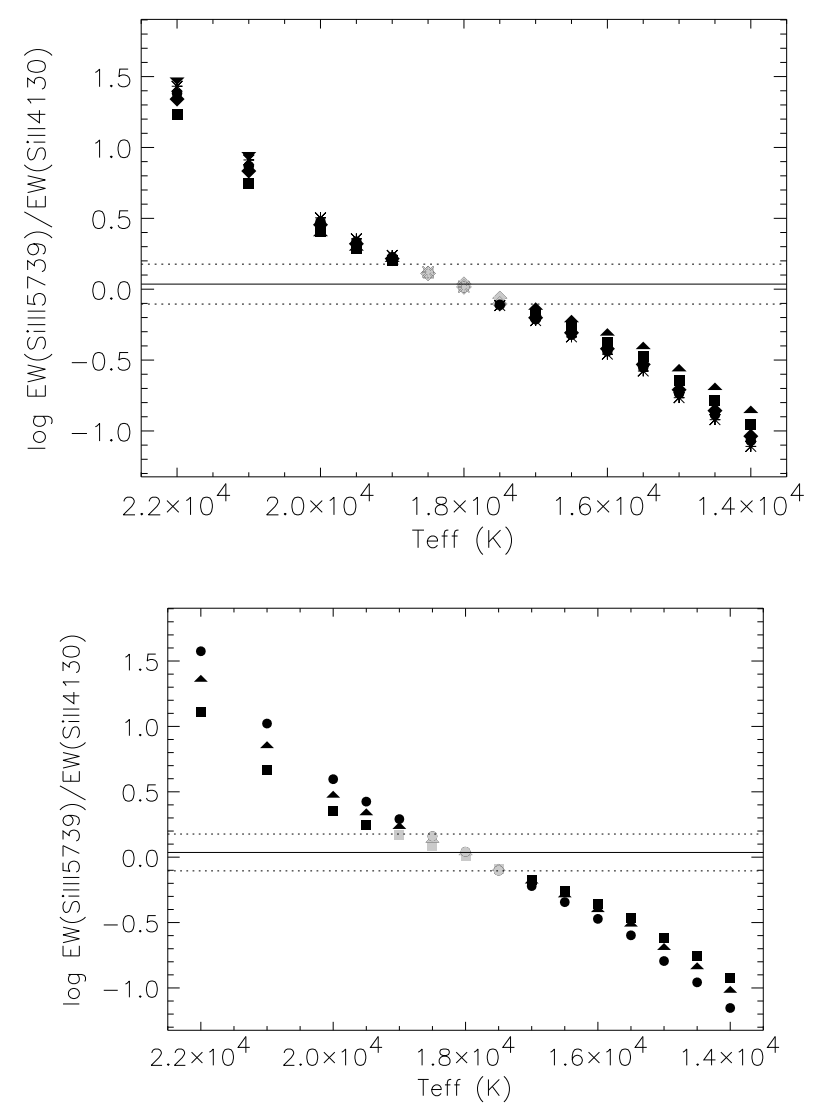

Fig. B.4. Synthetic simulation of the effect of the microturbulence (top) and the Si abundance (bottom) on the logarithmic EW ratios of Si lines of different ionization stages. This example gives the (synthetic) EW ratio of Si III 5739 to Si II 4130 against a selected range of effective temperatures, for a fixed surface gravity $(\log g=2.5)$ and wind parameters $\left(\dot{M}=0.144 \times 10^{-7} M_{\odot} / \mathrm{yr}, v_{\infty}=500 \mathrm{~km} \mathrm{~s}^{-1}, \beta=0.9\right)$. The ratios are evaluated for the different possibilities of the microturbulence, for a Si abundance fixed at -4.79 (upper panel: $\xi=3,6,10,12,15$, and $20 \mathrm{~km} \mathrm{~s}^{-1}$; triangle up, square, diamond, circle, asterisk, triangle down, respectively) and for the different possibilities of the Si abundances, for a microturbulence fixed at $10 \mathrm{~km} \mathrm{~s}^{-1}(\log n(\mathrm{Si}) / n(\mathrm{H})=-4.79,-4.49$, and -4.19: circle, triangle up, and square, respectively). The horizontal lines show the observed EW ratio and its corresponding uncertainty region. Acceptable EW ratios, which fall within these boundaries, are indicated in grey.

since the Si abundance affects all Si lines in the same direction. For each effective temperature point in the grid, the ratios of the observed equivalent widths of Si IV to Si III and/or Si III to Si II are compared to those obtained from the model grid, given a certain $\log g$ and wind parameters (see Fig. B.4). For each combination of Si IV/III and/or Si III/II lines, this results in a range of "acceptable" effective temperatures for which the observed EW ratio is reproduced, within the observed errors (indicated as grey symbols in Fig. B.4). From the different line ratios, slightly different temperatures may arise. Based on the assumption that $\log g$ and the wind parameters are known perfectly, the combination of all these possibilities constitutes a set of acceptable effective temperatures.

Once we have a list of acceptable temperatures, we can derive for each temperature the most suitable microturbulent velocity and $\mathrm{Si}$ abundance, by analyzing the variation in equivalent 

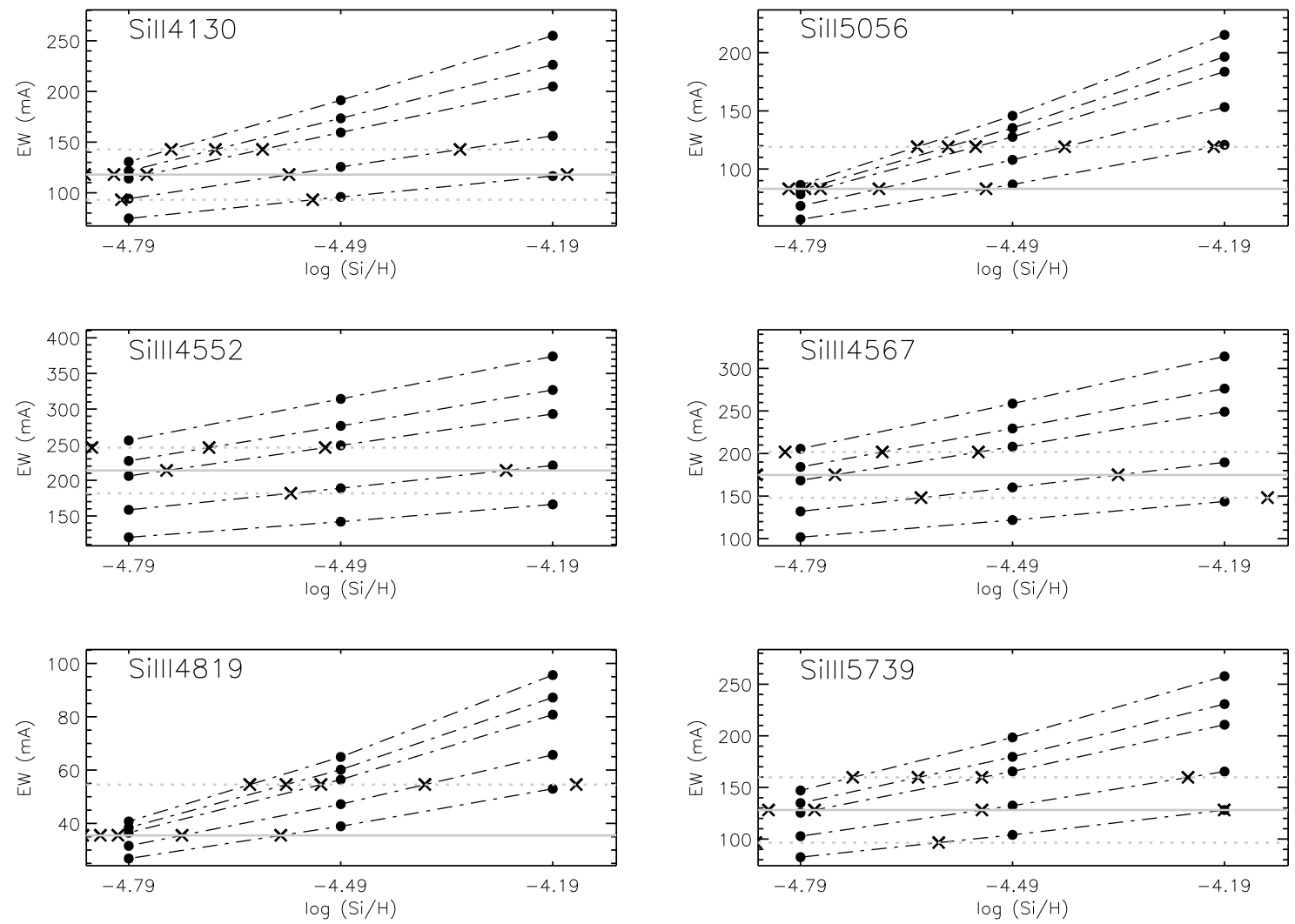

Fig. B.5. Step 1 (for each $T_{\text {eff }}$ and for each line): For each of the five considered microturbulent velocities (5 dash-dotted lines, from low EW to high EW: 3, 6, 10,12,15 $\mathrm{km} \mathrm{s}^{-1}$ ), we derive the Si abundance that reproduces the observed EW (horizontal grey lines) of the spectral line by linear interpolation. The Si abundances and the corresponding upper and lower limit are represented by the crosses. The displayed example is a synthetic simulation. Only a selection of lines is shown. Usually many more lines can be used. In this example, no Si IV was "observed", so only Si II and Si III were considered.

width of different lines as a function of these quantities. In what follows, we describe the full process, step by step:

\section{- Step 1: Deriving the abundances for each microturbulence,} line by line

In this first step, the observed EW of each Si line is compared to the set of theoretically predicted EWs corresponding to the available combinations of $\mathrm{Si}$ abundance and microturbulence in the grid (see Fig. B.5). For each microturbulent velocity (and for each Si line), we search for the range of $\mathrm{Si}$ abundances that reproduce the observed EW, within the observed errors. This is performed by linear interpolation. We note that this provides only an approximative estimate of the Si abundance. Indeed, as can be seen in Fig. B.5, the change in EW with increasing microturbulence is marginal for weak lines, but increases for stronger spectral lines, indicating that we may no longer be in the linear part of the curve of growth.

- Step 2: Deriving the mean abundance for each microturbulence and the slope of the best fit

In a second step, we compare, for each microturbulence, the abundances (with the derived uncertainties) found in step 1 to the observed EW of each line (see Fig. B.6). Since a star can have only one Si abundance, we should infer the same $\mathrm{Si}$ abundance from all the different $\mathrm{Si}$ lines, i.e., the slope of the best-fit relation to all the lines should be zero. From the different abundances derived from each line, we can calculate the mean abundance for each value of the microturbulence.

- Step 3: Deriving the "interpolated" microturbulent velocity In a third step, we investigate the change in slope when varying the microturbulence (see upper panel Fig. B.7). By linear interpolation, we find the microturbulence for which the slope would be zero (i.e., for which the Si abundance derived from each line separately would be the same). This is the estimated "interpolated" microturbulence.

- Step 4: Deriving the "interpolated" abundance

Using now the relation between the microturbulence and the mean abundance derived in step 2, we interpolate to find the estimated "interpolated" Si abundance at the estimated "interpolated" microturbulence found in step 3 (see lower panel Fig. B.7).

- Step 5: Deriving the "interpolated" effective temperature After obtaining the estimated "interpolated" values of the microturbulence and $\mathrm{Si}$ abundance, we can return to Fig. B.4 and interpolate in two dimensions to derive a value of the effective temperature, which reproduces the observed EW. The mean of the effective temperatures from each line ratio is then accepted as the value of the "interpolated" effective temperature of the star.

- Step 6: Determination of the "closest" grid values

For the subsequent steps in the automatic procedure, we need the closest grid values to these estimated "interpolated" values rather than the true values themselves. These closest values constitute a new entry in the list of possibilities. If the true value of the Si abundance or microturbulence falls exactly between two grid points, both grid values are added to the list.

We repeat these steps for each "possible" grid temperature, initially derived from Fig. B.4 (grey symbols), and in this way obtain a set of new possible solutions that optimally reproduce 

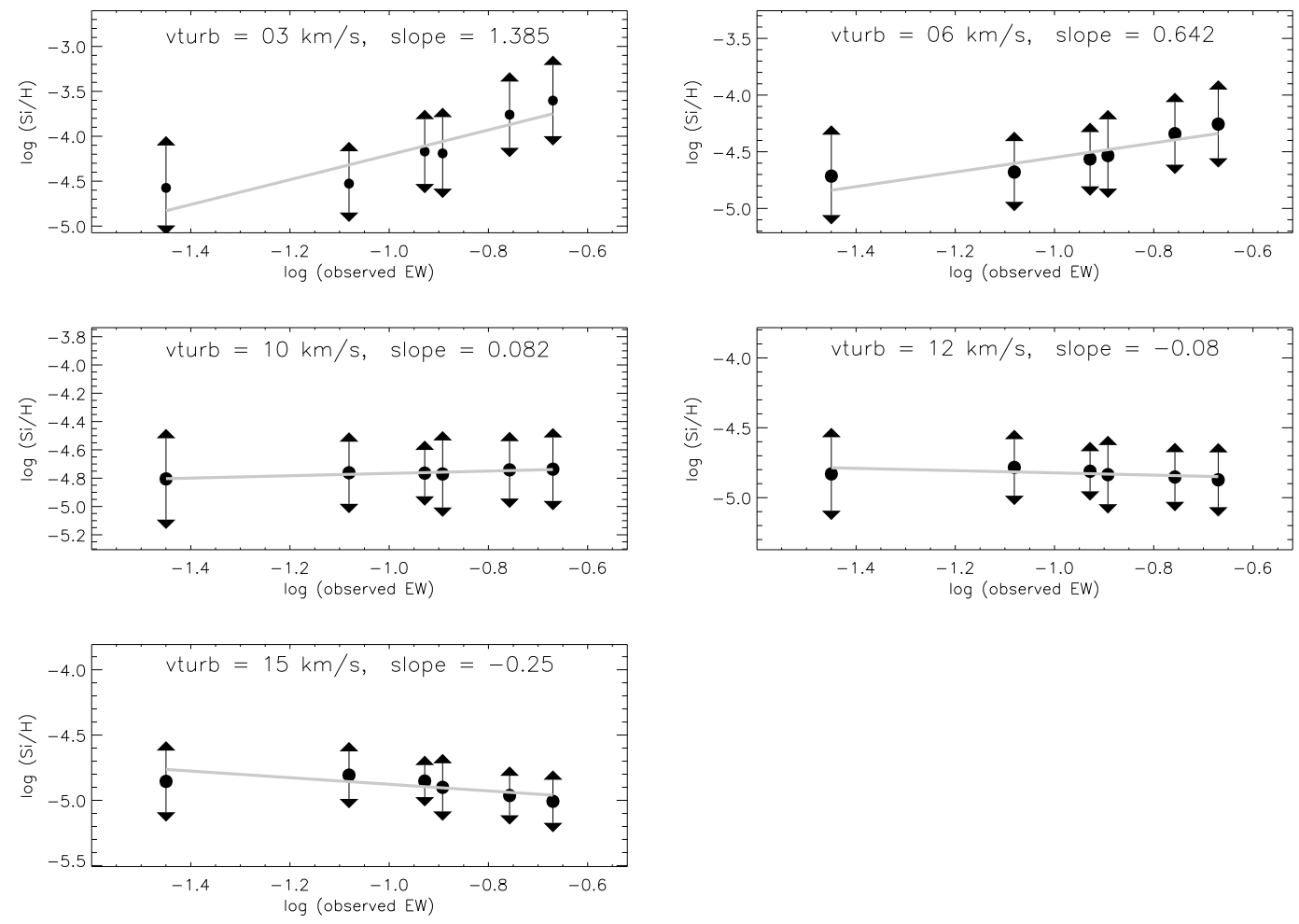

Fig. B.6. Step 2 (for each microturbulence): we plot the Si abundances and their uncertainties for all 6 lines for which we derived values in step 1, as a function of the observed EW. The least squares fit to the abundances are shown in grey. The microturbulent velocity for which the slope of this fit is zero (i.e., equal abundance from each line) gives an estimate of the "interpolated" microturbulent velocity. The displayed example is a synthetic simulation.

the $\mathrm{Si}$ lines. By temporarily fixing the values of $T_{\mathrm{eff}}, \xi$, and $\log n(\mathrm{Si}) / n(\mathrm{H})$ in the way described, we obtain a new estimate of the $\mathrm{He}$ abundance, denoted as $n(\mathrm{He}) / n(\mathrm{H})$. As for the $\mathrm{Si}$ abundance, we only consider three different values. We apply a method similar to that used to determine the Si abundance, in the sense that we derive the most-suitable abundance from each line separately, and take the mean value as the "interpolated" value. Figs. B.8 and B.9 illustrate this process. We note that we intrinsically assume that the microturbulence is the same throughout the atmosphere, i.e., that there is no radial stratification. In this way, the microturbulence, necessary to account for the broadening of the He lines, can be assumed to be the same as that derived from the Si lines. We note also that the uncertainty in the derived $\mathrm{He}$ abundance will be larger when the He abundance is lower than 0.10 . In this case, we are indeed forced to extrapolate to a region where it is unclear how the dependence of the equivalent width on abundance will change. The decrease towards abundances lower than solar may be steeper or follow a logarithmic trend, in which case the predicted values would be underestimated. In this case, we can only state that the solar abundance is an upper limit to the true abundance. Values lower than the primordial He abundance of $\sim 0.10$ can no longer be considered physical, except when diffusion effects begin to play a role and He settles. In that case, the He abundance observed at the stellar surface could be lower. These chemically peculiar stars are referred to as He weak stars and are usually high-gravity objects. Helium settling was not taken into account in our models.

Method 2: When data of only one ionization stage of $\mathrm{Si}$ is available. (Mostly for late B-type stars, for which we only have Si II, but also for a few hotter objects around $T_{\text {eff }}=23000 \mathrm{~K} \pm$ $3000 \mathrm{~K}$ with Si III) only, we can no longer use the ionization
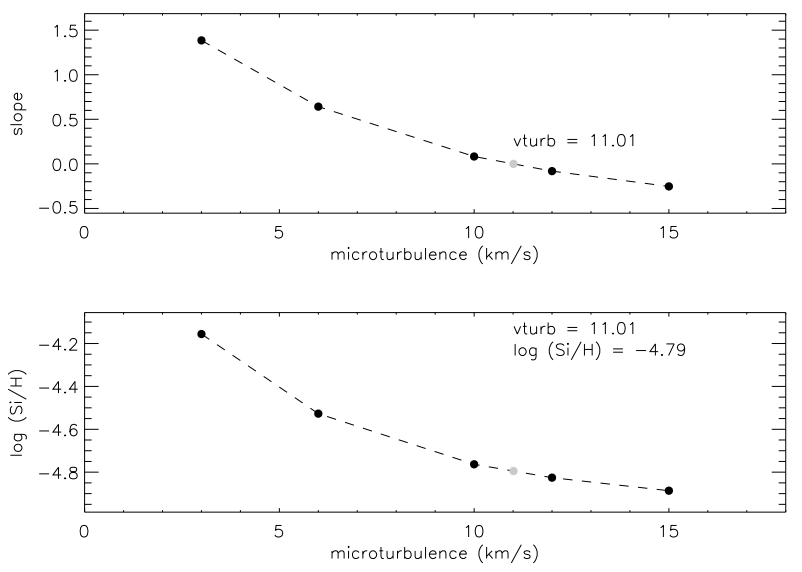

Fig. B.7. Steps 3 \& 4: the "interpolated" microturbulent velocity and the accompanying "interpolated" Si abundance (indicated in grey) are derived from the position where the slope of the best fit (step 2) is zero. The Si abundances, given in the lower panel, are the mean abundances derived in step 2 . The displayed example is a synthetic simulation.

balance to derive information about the effective temperature, independent of the abundances of $\mathrm{He}$ and $\mathrm{Si}$, and independent of the microturbulence. Indeed, we have only two known factors (in the case of late B-type stars: EW of He I and EW of $\mathrm{Si}$ II) to derive four unknown parameters $\left(T_{\mathrm{eff}}, \xi, \log n(\mathrm{Si}) / n(\mathrm{H})\right.$, $n(\mathrm{He}) / n(\mathrm{H}))$. Fortunately, we can constrain the effective temperature quite well. For instance, in the cold B-type domain, He I is very sensitive to changes in effective temperature (see, e.g., Fig. 2 in Lefever et al. 2007a), which means that we can use the joint predictive power of He I and Si II to derive a set of plausible values of $T_{\text {eff }}$. This is achieved by comparing the theoretical 
K. Lefever et al.: Fundamental parameters of B stars in the FoV of CoRoT
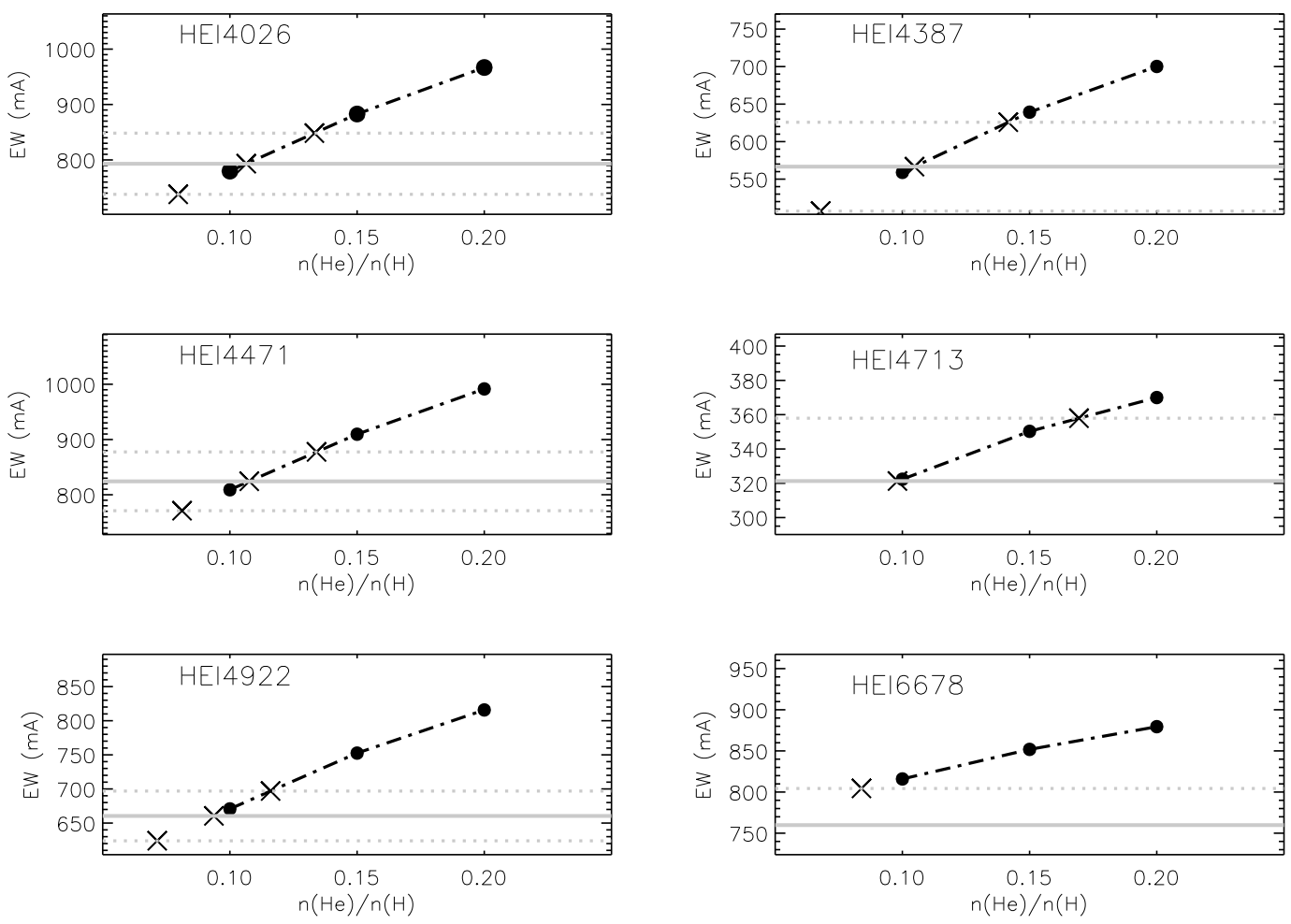

Fig. B.8. For each He line, the most suited He abundance is derived through interpolation. The theoretically predicted values are shown as filled circles, the interpolated values as crosses. The horizontal lines indicate the observed equivalent width and the observed errors. The displayed example is a synthetic simulation.

EWs of all combinations of Si abundance, microturbulence, and effective temperature (within a range $\pm 4000 \mathrm{~K}$ around the currently investigated $T_{\text {eff }}$ ) with the observed EWs, and selecting the "best" combinations from an appropriate log-likelihood function (for details, see Lefever 2007). This method is not only applicable to the cool domain, but also for those hotter objects where only Si III is present, in this case because the EW of Si III varies far more significantly with $T_{\text {eff }}$ than the EW of He I.

For each of the derived plausible $T_{\text {eff }}$ values, we need to identify the corresponding $\mathrm{Si}$ (from the Si lines) and He abundances (from the He lines) and the microturbulence (from the $\mathrm{Si}$ and/or He lines). Since the He lines are affected by Stark broadening, we use only Si lines to derive the microturbulent broadening. This means that we have to derive only three unknown parameters from data of two known parameters: two from the Si lines $(\log n(\mathrm{Si}) / n(\mathrm{H})$ and $\xi)$, and one more from the He lines $(n(\mathrm{He}) / n(\mathrm{H})$, assuming that the microturbulence in $\mathrm{Si}$ and $\mathrm{He}$ is similar), which still leaves us with one free parameter that we have to assume to be able to fix the other two. We chose to "fix" the Si abundance, since it has no direct influence on the He lines. However, we do consider each of the three possibilities for the $\mathrm{Si}$ abundance in the grid, i.e., we consecutively consider the three cases in which $\mathrm{Si}$ is depleted, solar, and enhanced. This also allows us to determine the microturbulent velocity, which is consequently used to fix the He abundance from the He lines.

To finally decide which of the various combinations matches the observations the most closely, a twofold check is applied: (i) all profile-sets are inspected by eye (this, again, excludes a fully automatic procedure), since parameter combinations leading to obviously poor fits can be clearly excluded on this basis; (ii) a log-likelihood procedure is again used to find the most closely matching model among the list of final solutions, combining the likelihoods of the EWs of the $\mathrm{Si} / \mathrm{He}$ lines and the profile shapes of all lines, respectively (see Lefever 2007).

Even though, with this procedure, we are able to derive reasonably good estimates of most of the stellar/wind parameters, we have no means of restricting the $\mathrm{Si}$ abundance in a reliable way as it is possible within "method 1". The outcome of maximizing the log-likelihood can only give us a general idea of the abundance, but still leaves us with a larger uncertainty, which has been accounted for in the adopted error budget (see Table 3).

\section{B.4. Macroturbulence $v_{\text {macro }}$}

The macroturbulence considered is characterized by a radialtangential model (Gray 1975). In this model, a time-independent velocity field is assumed to be active and modeled by assuming that a fraction $\left(A_{\mathrm{r}}\right)$ of the material is moving radially while the complementary fraction moves tangentially $\left(A_{t}\right)$. In our model for $v_{\text {macro }}$, we use the standard assumption that the radial and the tangential components are equal proportionally. Assuming in addition that the distribution of the velocities in the radial and the tangential directions are Gaussian, the effect of macroturbulence can be introduced in the synthetic line profiles by means of a convolution with a function, which consists of a Gaussian and an additional term proportional to the error function (see Eq. (4) in Gray 1975). It changes the shape of the line profile, but leaves the equivalent width untouched.

Only weak lines should be used to determine $v_{\text {macro }}$. For B-type stars, Si lines are the most suitable, since they most obviously show the presence of $v_{\text {macro }}$ in their wings. The user decides which Si lines should be used throughout the full process. To determine the strength of $v_{\text {macro }}$, we convolve the Si profiles of the (at that instant) best-fit model with different values of 


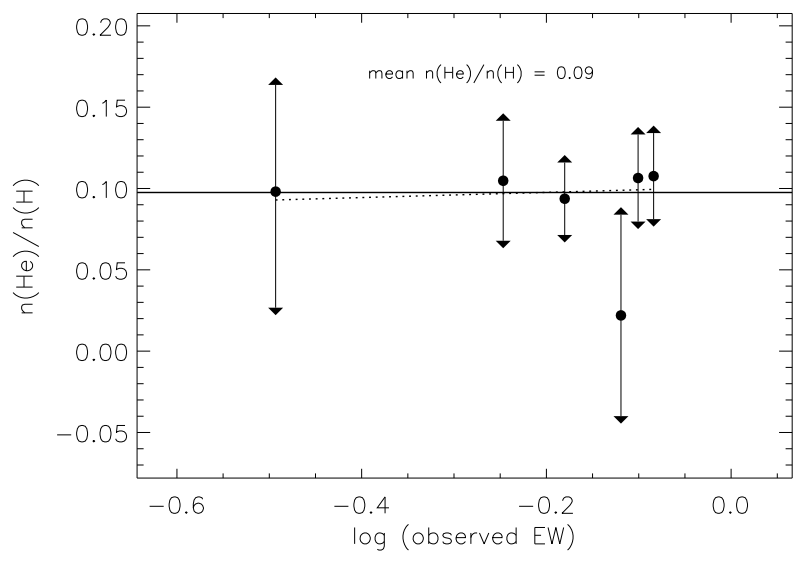

Fig. B.9. The finally provided He abundance is calculated as the mean over all lines. The abundance derived from each line separately is represented by the filled circles, while the arrows show the derived errors. The best linear fit is indicated as the dotted line, while the horizontal solid line shows the mean value. The displayed example is a synthetic simulation.

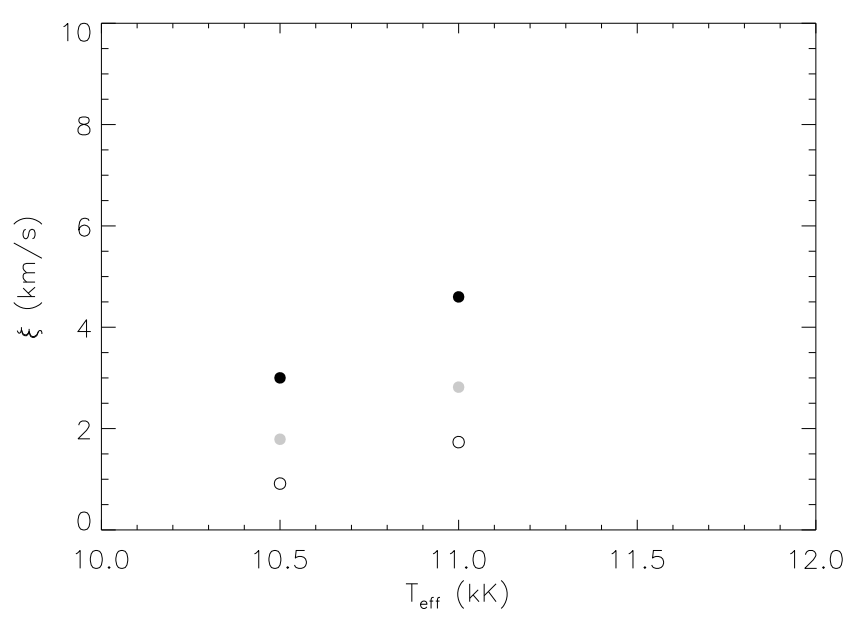

Fig. B.10. Once a set of possible effective temperatures is fixed from the joint predictive power of He I and Si II (see text), we can determine for each of these temperatures (in this case: 10500 and $11000 \mathrm{~K}$ ) the correct microturbulence, under the assumption that we know the $\mathrm{Si}$ abundance. Resulting combinations are shown for $\log n(\mathrm{Si}) / n(\mathrm{H})=-4.19$ (open circles), -4.49 (grey filled circles) and -4.79 (black filled circles). The displayed example is a synthetic simulation.

$v_{\text {macro }}{ }^{8}$. The consecutive values considered in the convolution are chosen using a bisection method, in initial steps of $10 \mathrm{~km} \mathrm{~s}^{-1}$. The bisection continues as long as the stepsize is greater than or equal to $0.1 \mathrm{~km} \mathrm{~s}^{-1}$, which will be the final precision of the $v_{\text {macro }}$ determination. For each considered $v_{\text {macro }}$-value, we compute its log-likelihood to quantify the difference between the obtained synthetic profile and the observed line profile, as follows:

$l \equiv \sum_{i=1}^{n}\left[-\ln (\sigma)-\ln (\sqrt{2 \pi})-\frac{1}{2}\left(\frac{y_{i}-\mu_{i}}{\sigma}\right)^{2}\right]$,

where $n$ is the considered number of wavelength points within the line profile, $y_{i}$ is the flux at wavelength point $i$ of the observed line profile, $\mu_{i}$ is the flux at point $i$ of the synthetic profile (convolved with the $v_{\text {macro }}$ under consideration), and $\sigma$ is the noise, i.e., $1 / \mathrm{SNR}$, of the considered line profile. A thorough discussion

\footnotetext{
8 At this point, the profile has already been convolved with the appropriate rotational and instrumental profiles.
}
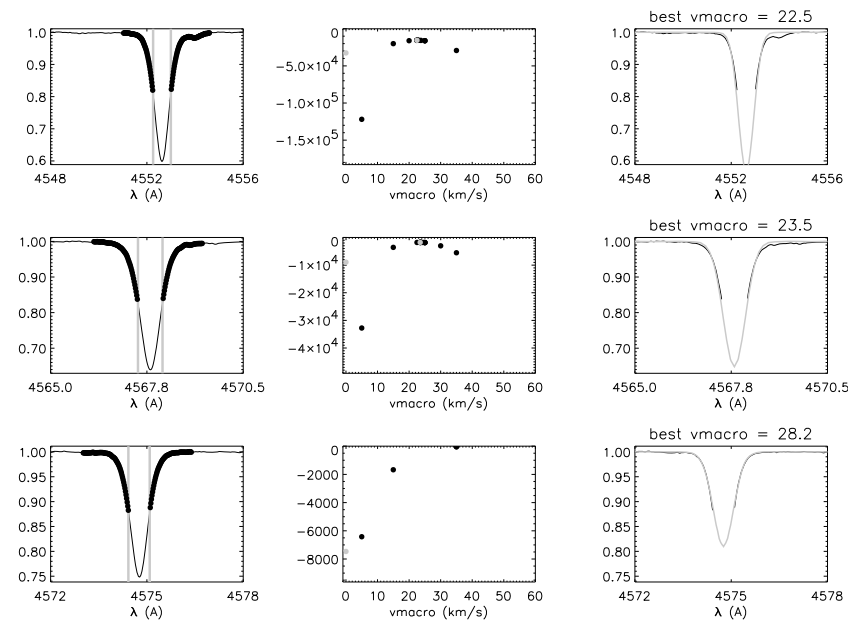

Fig. B.11. Determination of the macroturbulent velocity of $\beta \mathrm{CMa}$ for three different Si lines. The panels should be read from left to right as follows. Left: The left and right edge of the line region (i.e. outermost dots at continuum level) are indicated by the user at the start of the procedure. The blue and red edges (grey vertical lines) are determined as the point where the flux is half of the minimal intensity. They determine how far the wings extend towards the line center. The black dots represent the points that are finally used to determine the macroturbulence. Middle: obtained log-likelihood as a function of $v_{\text {macro }}$ (see Eq. (B.1)). Note the broad maximum in the log-likelihood distribution, which gives rather large error bars. Right: the fit with the best $v_{\text {macro }}$ is shown as the grey profile. Note that the significant amount of macroturbulence might be explained by the fact that this star exhibits non-radial oscillations (Mazumdar et al. 2006).

of why this particular likelihood function is suitable for spectral line fitting can be found, e.g., in Decin et al. (2007).

The same procedure is repeated for all considered line profiles, and the mean, derived from the different lines, infers the final $v_{\text {macro }}$. The error is set by the maximal deviation of the de-

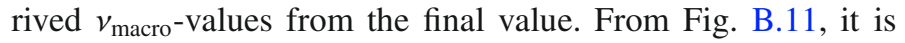
clear that the shape of the log-likelihood distribution close to the maximum can be quite broad, which indicates that the errors in $v_{\text {macro }}$ with respect to the log-likelihood are significant.

A warning is necessary here. Aerts et al. (2009) showed that time-dependent line profile variations due to non-radial gravitymode oscillations, which are expected in B stars, offer a natural physical explanation of macroturbulence. These oscillations affect the estimate of $v \sin i$, both from the Fourier method and from a goodness-of-fit approach. The only way to assess the effect of oscillations on the $v \sin i$ determination is by taking an appropriate time series of line profile variations (e.g., Aerts \& De Cat 2003). Luckily, an inappropriate $v \sin i$ estimate, accompanying a line profile fit in terms of $v_{\text {macro }}$, does not have a serious effect on the determination of the other parameters of the star from snapshot spectra, such that the approach implemented in AnalyseBstar is valid as long as the values of $v \sin i$ and $v_{\text {macro }}$ are not used for physical interpretations. A large value of the macroturbulence is an indication that time-dependent pulsational broadening may occur in the star being investigated.

\section{B.5. Surface gravity $\log g$}

The surface gravity $\log g$ is the result of fitting the Balmer lines. We mainly use $\mathrm{H} \gamma$ and $\mathrm{H} \delta$, possibly complemented with $\mathrm{H} \beta$ in the case of weak winds (i.e., if $\log Q \leq-13.80$ ). Since $\mathrm{H} \epsilon$ is somewhat blended, and the merging is not always reliable for 
this region (see above), this line is not used as a primary gravity determinator, but only serves as an additional check (together with $\mathrm{H} \beta$, in the case of stronger winds). Since we wish to define the profile only from the extreme wings down to the strongest curvature (see discussion of the different contributions below), we exclude the central part of the line, accounting for several mechanisms, that affect the core of the Balmer lines to some extent, e.g., rotation, micro- and macroturbulence and thermal broadening. Once we have removed the inner part of the line profile, we compare the observed Balmer lines with the synthetic line profiles, by considering all possible values of $\log g$ available at this given effective temperature gridpoint. We decide which value of gravity is the best, by maximizing its log-likelihood (see Eq. (B.1)), calculated over all Balmer lines. With this procedure, we encountered the following problem: $\mathrm{H} \gamma$ and $\mathrm{H} \delta$ (our main gravity indicators) can sometimes be seriously affected by a large amount of blends. Ignoring them can lead to a serious overestimate of the gravity, since the log-likelihood function will take all these blends into account (see Fig. B.12). Therefore, we developed a procedure to find the "line continuum", by cutting away the blends. The procedure is basically a sigma-clipping algorithm that retains only those points that have a flux higher than both neighboring points (the so-called "high points") and where, at the same time, the difference is less than 1/SNR. After removing all other flux points, only the "line continuum" is left (see middle panel Fig. B.12). We interpolate this continuum to obtain the flux at all original wavelength points, and the inner part of the line is added again (i.e., the part between the last blend in the blue wing and the first blend in the red wing, but obviously still without the central core). All original flux points that deviate by less than 1/SNR from the interpolated "line continuum" are also included again. In this way, we keep only the flux points that really determine the shape of the wing to fit the synthetic Balmer wings, and we are able to determine an accurate value of $\log g$ (see right panel Fig. B.12). We realize that some points that may be marked as local continuum in this way, may still be lower than the true local continuum level, because of the transition of one blend into another. However, this will only be the case for very few points, which will have no significant weight in the log-likelihood.

The finally accepted surface gravity is the value of $\log g$ that provides the best fit to all selected Balmer lines simultaneously. Half the gridstep in $\log g$ could thus be considered a good error estimate. However, to account for the coupling with $T_{\text {eff }}$, we consider 0.1 dex as a more appropriate error. This gravity should be corrected for centrifugal acceleration $\left(\rightarrow \log g_{c}\right)$ due to stellar rotation by a factor $(v \sin i)^{2} / R_{*}$, when calculating, e.g., the mass of a star (Repolust et al. 2005, and references therein).

\section{B.6. Wind parameters}

A change in mass-loss rate will mainly affect the shape of $\mathrm{H} \alpha$. For cool objects and weak winds, $\mathrm{H} \alpha$ is nearly "photospheric" and will, in essence, be an absorption profile, with more or less symmetric components, since the "re-filling" by the wind is low. In the case of stronger winds, $\mathrm{H} \alpha$ will take the shape of a typical P Cygni profile. For hot objects, where $\mathrm{H} \alpha$ is dominated by recombination processes, and high mass-loss rates, the profile may even appear in full emission.

The wind parameter $\beta$ determines the velocity law, which directly influences the density. The red wing of $\mathrm{H} \alpha$ is well suited to determining $\beta$, since it is formed by emission processes alone, averaged over the receding part of the (almost) complete wind. For a fixed mass-loss, a "slower" velocity law (i.e., a higher $\beta$

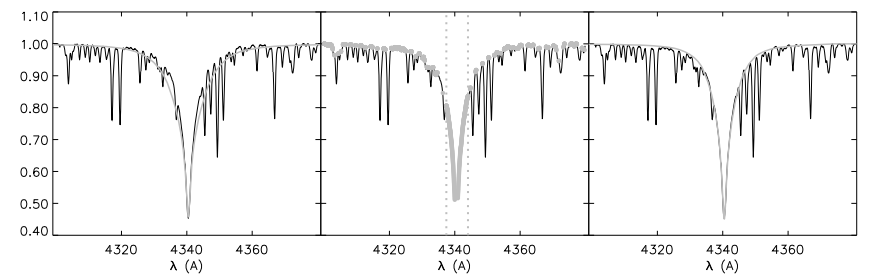

Fig. B.12. Illustration of the effect of blends on the determination of $\log g$, and the clipping algorithm to improve the fit, for $\beta$ CMa. Left: the many blends in the wings of the profile prevent an accurate least squares fit and lead to too broad wings, implying too high a gravity. Central: from the observed line profile (black), only the "high points" (grey dots), which deviate less than 1/SNR from their neighbors, are kept, complemented with the inner part of the line between the last blend in the blue wing and the first blend in the red wing (indicated as grey vertical lines), but still excluding the central core, which is affected by several broadening mechanisms. Right: after the removal of all blends through sigma clipping (see text), we are able to obtain a good representation of the line wings (grey profile) and, therefore, to derive a very accurate value for the gravity.
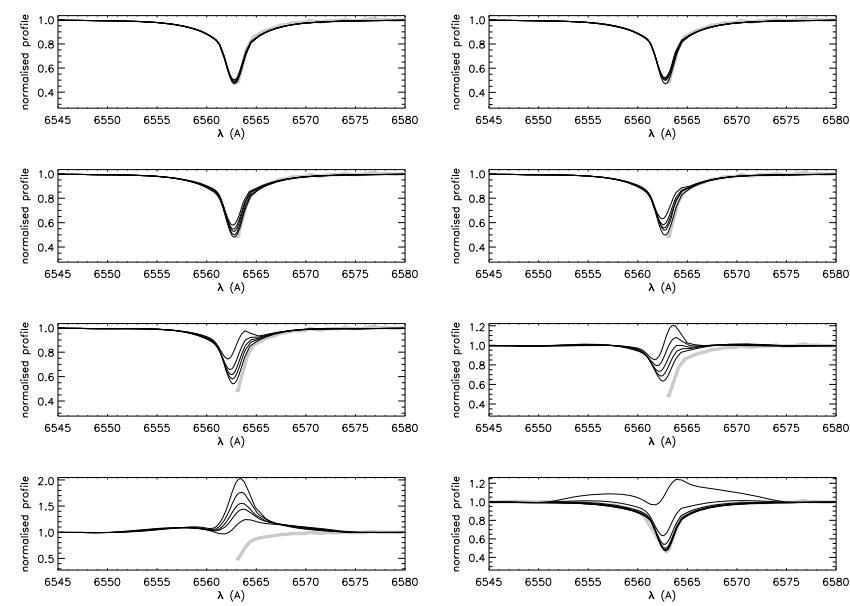

Fig. B.13. (Synthetic simulation) For each different wind strength parameter $\log Q$ (7 values, from left to right and from top to bottom: $\log Q=-14.30,-14.00,-13.80,-13.60,-13.40,-13.15,-12.70)$, we search for the best $\beta$ (in each panel, the 5 different values for $\beta$ are indicated: $0.9,1.2,1.5,2.0,3.0)$ by comparing only the red wing (grey part of the profile). Then the synthetic profiles of each best $(\log Q, \beta)$ combination are compared to the entire $\mathrm{H} \alpha$ profile (bottom right).

value) will result in higher densities in the lower atmosphere, close to the star. This enlarges the number of emitted photons with velocities close to the line center, resulting in more emission. Around the central wavelength, the absorption component of the line profile refills and the emission component becomes stronger. Therefore, the slope of the red wing of the P Cygni profile becomes steeper. In this sense, the steepness of the red wing is a measure of the value of $\beta$.

We estimate the wind parameters $\log Q$ and $\beta$ by comparing the observed $\mathrm{H} \alpha$ profile with the different synthetic $\mathrm{H} \alpha$ profiles by making combinations of $\log Q$ and $\beta$. We decided to ensure that the determination of the wind parameters was not too sophisticated, by simply using the best-fit values for $\log Q$ and $\beta$ available in the grid, without interpolation and further refinement. Once we have selected for each $\log Q$ the most suitable $\beta$, we decide on the best combination by considering the best fit to the entire profile. 


\section{B.7. Some final remarks}

At the end of this iteration cycle, an acceptance test is performed. When the method can run through the entire cycle without any of the fit parameters needing to be updated, the model is accepted as a good model, and obtains the flag " 2 ". If a closer fitting model was found (i.e., when one or more parameters are changed), the initial model is rejected and receives flag " 0 ", whereas the improved model is added to the list of possibilities, and receives flag " 1 " (cf. "models to check" in the flowchart diagram, Fig. B.1). As long as the list with possibilities with flag "1" is not empty, the parameters for the next possibility are taken as new starting values.

Line profile fits are always performed allowing for a small shift in wavelength, or radial velocity ( 5 wavelength points in either direction, corresponding to typically $5 \mathrm{~km} \mathrm{~s}^{-1}$ ), to prevent flux differences adding up in the case of a small radial velocity displacement.

\section{B.8. The underlying FASTWIND BSTAR06 grid}

Even though the majority of the CoRoT targets consisted of late B-type stars, we nevertheless invested in a grid that covers the complete parameter space of B-type stars. In this way, we hoped to establish a good starting point for future (follow-up) detailed spectroscopic analysis of massive stars. The grid has been constructed to be as representative and dense as possible to provide a wide variety of stellar properties within a reasonable computation time. Hereafter, we give a short summary of the considered parameters. For a more detailed description, we refer to Lefever et al. (2007a):

- 33 effective temperature ( $T_{\text {eff }}$ ): from $10000 \mathrm{~K}$ to $32000 \mathrm{~K}$, in steps of $500 \mathrm{~K}$ below $20000 \mathrm{~K}$ and in steps of $1000 \mathrm{~K}$ above it.

- On average 28 surface gravities $(\log g)$ at each effective temperature point: from $\log g=4.5$ down to $80 \%$ of the Eddington limit.

- One "typical" value of radius $\left(R_{*}\right)$ for each $\left(T_{\text {eff }}, \log g\right)$ gridpoint: approximate value obtained by interpolating between evolutionary tracks, recalling that a rescaling to the "real" value is required when analyzing specific objects. Indeed, the real radius can then, in most cases, be determined from the visual magnitude, the distance of the star and its reddening, and from the theoretical fluxes of the best-fit model.

- Three values to fix the chemical composition: $n(\mathrm{He}) / n(\mathrm{H})=$ $0.10,0.15$, and 0.20 , and $\log n(\mathrm{Si}) / n(\mathrm{H})=-4.19,-4.49$, and -4.79 (i.e., enhanced, solar, and depleted). The background elements (responsible, e.g., for radiation pressure and lineblanketing) were assumed to be of solar composition.

- Seven values of the wind-strength parameter $\log Q$.

- One "typical" value of the terminal wind velocity $\left(v_{\infty}\right)$ : estimated from the relation between observed terminal velocity and photospheric escape velocity (Kudritzki \& Puls 2000, and references therein), where the latter quantity had been calculated from the actual grid parameters $\left(\log g, R_{*}\right.$ and $\left.T_{\text {eff }}\right)$.

\section{References}

Aerts, C. 2000, A\&A, 361, 245

Aerts, C. 2008, in IAU Symp., 250, 237
Aerts, C., \& De Cat, P. 2003, Space Sci. Rev., 105, 453

Aerts, C., Puls, J., Godart, M., et al. 2009, A\&A, 508, 409

Briquet, M., \& Morel, T. 2007, Commun. Asteroseismol., 150, 183

Butler, K., \& Giddings, J. R. 1985, in Newsletter on Analysis of Astronomical Spectra, No. 9, Univ. London

Crowther, P. A. 1998, in Fundamental Stellar Properties, ed. T. R. Bedding, A. J. Booth, \& J. Davis, IAU Symp., 189, 137

Decin, L., Hony, S., de Koter, A., et al. 2007, A\&A, 475, 233

Desmet, M., Briquet, M., Thoul, A., et al. 2009, MNRAS, 396, 1460

Dufton, P. L., Smartt, S. J., Lee, J. K., et al. 2006, A\&A, 457, 265

Dupret, M., De Ridder, J., Neuforge, C., Aerts, C., \& Scuflaire, R. 2002, A\&A, 385, 563

Evans, C. J., Smartt, S. J., Lee, J.-K., et al. 2005, A\&A, 437, 467

Frémat, Y., Neiner, C., Hubert, A.-M., et al. 2006, A\&A, 451, 1053

Fridlund, M., Baglin, A., Lochard, J., et al. 2006, The CoRoT Mission - Pre-

Launch Status - Stellar Seismology and Planet Finding, ESA SP, 1306

Giddings, J. 1981, Ph.D. Thesis, University of London, UK

Gräfener, G., Koesterke, L., \& Hamann, W.-R. 2002, A\&A, 387, 244

Gray, D. F. 1973, ApJ, 184, 461

Gray, D. F. 1975, ApJ, 202, 148

Gray, D. F. 1976, The observation and analysis of stellar photospheres (New York: Wiley-Interscience)

Handler, G., Jerzykiewicz, M., Rodríguez, E., et al. 2006, MNRAS, 365, 327

Hauschildt, P., \& Baron, E. 1999, J. Comp. Appl. Math., 109, 41

Hempel, M., \& Holweger, H. 2003, A\&A, 408, 1065

Hillier, D. J., \& Miller, D. L. 1998, ApJ, 496, 407

Hubeny, I., \& Lanz, T. 2000, BAAS, 32, 1531

Hubrig, S., Briquet, M., Schöller, M., et al. 2006, MNRAS, 369, L61

Hubrig, S., Briquet, M., Morel, T., et al. 2008, A\&A, 488, 287

Hunter, I., Dufton, P. L., Smartt, S. J., et al. 2007, A\&A, 466, 277

Hunter, I., Brott, I., Lennon, D. J., et al. 2008a, ApJ, 676, L29

Hunter, I., Lennon, D. J., Dufton, P. L., et al. 2008b, A\&A, 479, 541

Kudritzki, R., \& Puls, J. 2000, ARA\&A, 38, 613

Kurucz, R. L. 1993, CD-ROM 13 (Cambridge: SAO)

Lefever, K. 2007, Ph.D. Thesis, Institute of Astronomy, K.U. Leuven, Belgium

Lefever, K., Puls, J., \& Aerts, C. 2007a, in The Future of Photometric,

Spectrophotometric and Polarimetric Standardization, ed. C. Sterken, ASP Conf. Ser., 364, 545

Lefever, K., Puls, J., \& Aerts, C. 2007b, A\&A, 463, 1093

Levenberg, K. 1944, The Quarterly of Applied Mathematics, 2, 164

Maeder, A., \& Meynet, G. 2000, ARA\&A, 38, 143

Markova, N., \& Puls, J. 2008, A\&A, 478, 823

Marquardt, D. 1963, SIAM J. Appl. Math., 11, 431

Massey, P., Zangari, A. M., Morrell, N. I., et al. 2009, ApJ, 692, 618

Mazumdar, A., Briquet, M., Desmet, M., et al. 2006, A\&A, 459, 589

Miglio, A., Montalbán, J., \& Dupret, M.-A. 2007a, MNRAS, 375, L21

Miglio, A., Montalbán, J., \& Dupret, M.-A. 2007b, Commun. Asteroseismol., 151,48

Mokiem, M. R., de Koter, A., Puls, J., et al. 2005, A\&A, 441, 711

Morel, T., \& Aerts, C. 2007, Commun. Asteroseismol., 150, 201

Morel, T., Butler, K., Aerts, C., Neiner, C., \& Briquet, M. 2006, A\&A, 457, 651

Morel, T., Butler, K., Aerts, C., Neiner, C., \& Briquet, M. 2007, Commun. Asteroseismol., 150, 199

Morel, T., Hubrig, S., \& Briquet, M. 2008, A\&A, 481, 453

Neiner, C., Hubert, A.-M., \& Catala, C. 2005, ApJS, 156, 237

Niemczura, E., Morel, T., \& Aerts, C. 2009, A\&A, 506, 213

Pauldrach, A. W. A., Hoffmann, T. L., \& Lennon, M. 2001, A\&A, 375, 161

Prialnik, D. 2000, An Introduction to the Theory of Stellar Structure and Evolution (Cambridge, UK: Cambridge University Press)

Przybilla, N., Nieva, M.-F., \& Butler, K. 2008, ApJ, 688, L103

Puls, J., Urbaneja, M. A., Venero, R., et al. 2005, A\&A, 435, 669

Puls, J., Markova, N., Scuderi, S., et al. 2006, A\&A, 454, 625

Repolust, T., Puls, J., Hanson, M. M., Kudritzki, R.-P., \& Mokiem, M. R. 2005, A\&A, 440, 261

Saio, H., Kuschnig, R., Gautschy, A., et al. 2006, ApJ, 650, 1111

Santolaya-Rey, A. E., Puls, J., \& Herrero, A. 1997, A\&A, 323, 488

Scuflaire, R., Théado, S., Montalbán, J., et al. 2008, Ap\&SS, 316, 83

Simón-Díaz, S., \& Herrero, A. 2007, A\&A, 468, 1063

Solano, E., Catala, C., Garrido, R., et al. 2005, AJ, 129, 547

Trundle, C., Dufton, P. L., Hunter, I., et al. 2007, A\&A, 471, 625

Urbaneja, M. A. 2004, Ph.D. Thesis, Instituto de Astrofisica de Canarias, Spain

Vrancken, M., Lennon, D. J., Dufton, P. L., et al. 2000, A\&A, 358, 639 